\title{
Coordinated path following for unicycles: A nested invariant sets approach
}

\author{
Alireza Doosthoseini ${ }^{1}$, Christopher Nielsen \\ Department of Electrical and Computer Engineering, University of Waterloo, 200 University Ave. W, Waterloo, Ontario, N2L 3G1, Canada
}

\section{A R T I C L E I N F O}

\section{Article history:}

Received 3 July 2014

Received in revised form

11 April 2015

Accepted 8 June 2015

Available online 7 July 2015

\section{Keywords:}

Coordinated path following problem

Multi-agent path following manifold

Local coordination set

\begin{abstract}
A B S T R A C T
We formulate a coordinated path following problem for $N$ unicycle mobile robots as an instance of a nested set stabilization problem. Stabilization of the first set corresponds to driving the unicycles to their assigned paths. Stabilization of the second set, a subset of the first, corresponds to meeting the coordination specification. The first set is stabilized, for initial conditions sufficiently close to the set, in a decentralized manner using feedback linearization. For general coordination tasks we utilize feedback linearization to stabilize the nested set in a centralized manner, again, for initial conditions sufficiently close to the second set. In the special case in which coordination entails making the unicycles maintain a formation along their paths, we employ a semi-distributed control law under less restrictive communication assumptions. Experimental results are provided.
\end{abstract}

Crown Copyright @ 2015 Published by Elsevier Ltd. All rights reserved.

\section{Introduction}

Coordinated path following involves designing feedback controllers that make a group of agents each follow an output path while coordinating their motion. Coordinated motion along paths includes tasks like maintaining formations, traversing paths at a common speed and more general tasks like making the positions of some agents obey functional constraints that depend on the states of other agents. Coordinated path following is well-suited to applications in which accurate path traversal is vital and is motivated by applications in marine vehicle control (Encarnaçao \& Pascoal, 2001; Lapierre, Soetanto, \& Pascoal, 2004), search and rescue operations (Do \& Pan, 2007), and patrolling a pre-defined region (Zhang, Fratantoni, Paley, Lund, \& Leonard, 2007).

Studies on coordinated path following can be broadly classified by the manner in which the path following portion of the problem is solved. The most common approach is to parameterize each path, use the parameterization as a reference trajectory, and treat the evolution of the path parameter as an additional

\footnotetext{
The material in this paper was partially presented at the 52nd IEEE Conference on Decision and Control, December 10-13, 2013, Florence, Italy. This paper was recommended for publication in revised form by Associate Editor Hiroshi Ito under the direction of Editor Andrew R. Teel.

E-mail addresses: adoostho@uwaterloo.ca (A. Doosthoseini), cnielsen@uwaterloo.ca (C. Nielsen).

1 Tel.: +1 $5198884567 \times 37265$.
}

control input (Aguiar \& Hespanha, 2007; Dačić, Nešić, \& Kokotović, 2007; Ihle, Arcak, \& Fossen, 2007; Skjetne, Fossen, \& Kokotović, 2004). Linearization, Lyapunov-based methods, backstepping, and neural networks have been used to stabilize the associated error dynamics (Ghabcheloo, Pascoal, Silvestre, \& Kaminer, 2006, 2007; Ghommam \& Mnif, 2009; Wang, Wang, \& Peng, 2014; Xiang, Lapierre, Jouvencel, \& Parodi, 2009). Alternatively, in the curve extension method (Chen \& Tian, 2011; Paley, Leonard, \& Sepulchre, 2008; Zhang et al., 2007; Zhang \& Leonard, 2007), a smooth function, valid in a neighbourhood of each path, is employed such that the zero level set of this function is the desired path. Convergence to the path is achieved when the value of the smooth function reaches zero. A drawback of the aforementioned approaches is that invariance of the paths is not guaranteed independently of the coordination task. This is important because it ensures that even if coordination fails due to, say, communication errors, the individual robots remain on their paths.

In this paper, extending our preliminary work (Doosthoseini \& Nielsen, 2013), we solve a coordinated path following problem for a group of differential drive mobile robots modelled as dynamic unicycles. We characterize an embedded product submanifold in the state space of the unicycles called the multi-agent path following manifold. Convergence and invariance of the each unicycle's path is accomplished by making this set attractive and controlled invariant (El-Hawwary \& Maggiore, 2008; Nielsen, Fulford, \& Maggiore, 2010). We show that each unicycle is feedback equivalent, in a neighbourhood of its assigned path, to a system whose transversal and tangential dynamics to the path following 
manifold are both double integrators. This makes path following control design straightforward in comparison with other studies. While our results are local, valid in a neighbourhood of each unicycle's path, it is possible to extend the region of attraction of the proposed controllers using switched controllers (Tomlin \& Sastry, 1998).

The coordination aspect of the problem is typically enforced through clever re-parameterization of each system's assigned path. In Ghabcheloo et al. (2006) and Lapierre et al. (2004) each path is parameterized so that the desired formation corresponds to having each system's path parameter approach a common value. In Do and Pan (2007), Ihle et al. (2007) and Zhang, Lapierre, and Xiang (2013) a method called formation reference point is suggested to re-parameterize the assigned paths for each agent. The desired formation, which can change over time, is treated as a virtual geometric structure and a desired reference path is assigned to the centroid of the virtual structure. The reference path of the centroid determines the movement of the whole multi-agent system. The path of each agent is then re-parameterized according to its position in the virtual structure. A disadvantage of enforcing coordination via clever re-parameterization of the assigned paths is that the agents may leave their paths when the coordination task changes. Furthermore, most previous studies only consider position coordination, i.e., formation control, along the desired paths.

We do not rely on re-parameterization of the assigned paths. Instead, we model the coordination task as an embedded submanifold of the multi-agent path following manifold. Coordination is achieved if this submanifold is rendered attractive and controlled invariant. This viewpoint allows us to guarantee that when the robots are initialized on this submanifold, they remain coordinated indefinitely. It also allows one to change the coordination specification without causing the robots to leave their paths.

When all of the unicycles are assigned simple closed curves, our coordination problem becomes closely related to the problem of oscillator synchronization (Dörfler \& Bullo, 2014). In this case each unicycle's path following manifold is diffeomorphic to $\mathbb{S}^{1} \times \mathbb{R}$. An oscillator can be modelled as a double-integrator with state space $\mathbb{S}^{1} \times \mathbb{R}$. In this case coordinating the unicycle's velocities can be viewed as frequency synchronization of oscillators (Dorogovtsev, Goltsev, \& Mendes, 2008). When all the unicycles must have the same position along their paths, coordination can be viewed as a phase synchronization problem (Scardovi, Sarlette, \& Sepulchre, 2007). When all the unicycles are asked to maximally spread themselves along their closed-paths, the coordination task can be viewed as phase balancing (Sepulchre, Paley, \& Leonard, 2007).

Similarly, when all unicycles are assigned non-closed paths, coordination is closely related to consensus problem for double integrator dynamics. In this case each unicycle's path following manifold is diffeomorphic to $\mathbb{R}^{2}$. Consequently, in this special case, our approach allows one to use control laws in the literature that achieve consensus for double-integrators for our coordination tasks. For example, when coordination entails reaching consensus along paths, the results in Ren (2007) provide minimal connectedness conditions on the communication graph. When coordination involves reaching a common velocity along the paths the results in Chen, Wang, and Xiao (2009) provide control laws respecting a switching communication graph.

An advantage of our approach is that we allow for more general forms of coordination than position or velocity consensus or those discussed above. The penalty paid for allowing such a general class of coordination is that our controllers only provide a local solution and are generally centralized. However, one of the key advantages of the approach taken in this paper is that we are able to decouple the design of path following controllers from coordination controllers. This allows one to use, as discussed above, existing controllers from the literature in a modular fashion when more stringent communication constraints exist.

A preliminary version of this paper without the results in Sections 3.1 and 5.2, without Sections 5.3 and 5.4, and without the experimental results of Section 6 appeared in Doosthoseini and Nielsen (2013). In particular, the results in Section 5.2 contain proofs of various results omitted in Doosthoseini and Nielsen (2013).

\subsection{Notation}

Given $n \in \mathbb{N}, \mathbf{n}:=\{1, \ldots, n\}$. We denote the Euclidean inner product by $\langle x, y\rangle$ and the associated Euclidean norm by $\|x\|$. The point-to-set distance between $x \in \mathbb{R}^{n}$ and $A \subset \mathbb{R}^{n}$ is $\operatorname{dist}(x, A):=$ $\inf \{\|x-y\|: y \in A\}$. The symbols $\mathbf{I}_{n}$ and $\mathbf{0}_{n}$ represent, respectively, the $n \times n$ identity matrix and matrix of zeros while $1_{n}$ and $0_{n}$ represent the $n \times 1$ vector of ones and vector of zeros. Let $\mathbf{U}_{n}$ denote an $n \times n$ upper-triangular matrix with $u_{i j}=1, i \leq j, u_{i j}=0$, $i>j$. If $f: \mathbb{R}^{n} \rightarrow \mathbb{R}$ is a differentiable function, we denote by $\partial_{x_{i}} f$ its partial derivative with respect to $x_{i}$. The Jacobian of a $C^{1}$ map $f: \mathbb{R}^{n} \rightarrow \mathbb{R}^{m}$ evaluated at $p \in \mathbb{R}^{n}$ is written as $\mathrm{d} f(p)$. If $f: \mathbb{R}^{n} \rightarrow \mathbb{R}^{n}$ is a smooth vector field and $\phi: \mathbb{R}^{n} \rightarrow \mathbb{R}^{m}$ is a smooth map then $L_{f} \phi(x):=\langle\mathrm{d} \phi(x), f(x)\rangle$. If $f: \mathbb{R} \rightarrow \mathbb{R}^{n}$ then $f^{\prime}(\lambda):=\frac{\mathrm{d} f(\lambda)}{\mathrm{d} \lambda}$

\section{Coordinated path following problem}

Following El-Hawwary and Maggiore (2013), the model of unicycle $i, i \in \mathbf{N}$, is

$\dot{x}_{i}=v_{i} \cos \left(\theta_{i}\right)$
$\dot{y}_{i}=v_{i} \sin \left(\theta_{i}\right)$
$\dot{\theta}_{i}=u_{i, 2}$
$\dot{v}_{i}=u_{i, 1}$

where $\left(x_{i}, y_{i}\right)$ denotes the position of the unicycle in the plane, $\theta_{i}$ is the heading angle, and $v_{i}$ is the forward velocity of the unicycle. The control inputs $u_{i, 1}$ and $u_{i, 2}$ are, respectively, the forward acceleration and angular velocity. Let $\mathbf{x}_{i}:=\left(x_{i}, y_{i}, \theta_{i}, v_{i}\right) \in$ $\mathbb{R}^{2} \times \mathbb{S}^{1} \times \mathbb{R}$. Let $\tau\left(\theta_{i}\right):=\left(\cos \left(\theta_{i}\right), \sin \left(\theta_{i}\right)\right)$ denote the unicycle's heading. We take the position of the unicycle $i$ as its output $\mathbf{y}_{i}=$ $h_{i}\left(\mathbf{x}_{i}\right):=\left(x_{i}, y_{i}\right)$. The state of the multi-agent system is $\mathbf{x}:=$ $\left(\mathbf{x}_{1}, \ldots, \mathbf{x}_{N}\right) \in\left(\mathbb{R}^{2} \times \mathbb{S}^{1} \times \mathbb{R}\right)^{N}$.

\subsection{Communication}

We model communication between unicycles using a weighted directed graph $\mathscr{G}$ hereafter called the communication graph. Let $\mathrm{V}(\mathscr{G})=\left\{a_{1}, \ldots, a_{N}\right\}$ and $\mathrm{E}(\mathscr{G}) \subseteq \mathrm{V}(\mathscr{G}) \times \mathrm{V}(\mathscr{G})$ be, respectively, the vertex and edge set of $\mathscr{G}$. Each vertex represents an agent and an edge $\left(a_{i}, a_{j}\right)$ indicates that agent $j$ receives information from agent $i$. We denote by $w_{i j} \in \mathbb{R}$ the weight associated to edge $\left(a_{i}, a_{j}\right)$. For each vertex define its in-degree as $d_{\mathrm{in}}\left(a_{i}\right):=\sum_{\left(a_{j}, a_{i}\right) \in \mathrm{E}} w_{j i}$.

Definition 2.1. The in-degree matrix of $\mathscr{G}$ is $\Delta(\mathscr{G}):=\operatorname{diag}\left(d_{\text {in }}\left(a_{1}\right)\right.$, $\left.\ldots, d_{\text {in }}\left(a_{N}\right)\right)$. $\diamond$

Definition 2.2. The in-degree adjacency matrix of $\mathscr{G}$ is an $N \times N$ matrix whose ijth element is given by

$W(\mathscr{G})_{i, j}:= \begin{cases}w_{j i} & \text { if }\left(a_{j}, a_{i}\right) \in \mathrm{E}(\mathscr{G}) \\ 0 & \text { otherwise. } \diamond\end{cases}$

Definition 2.3. The weighed graph Laplacian of $\mathscr{G}$ is $L(\mathscr{G}):=$ $\Delta(\mathscr{G})-W(\mathscr{G}) . \diamond$ 
Definition 2.4. A directed graph $\mathscr{G}$ is rooted out-branching if it does not contain a directed cycle and there exists a vertex $a_{r} \in$ $\mathrm{V}(\mathscr{G})$ such that for all $a_{i} \in \mathrm{V}(\mathscr{G})$ there is a directed path from $a_{r}$ to $a_{i} . \diamond$

\subsection{Path following}

Each unicycle is assigned a path $\gamma_{i} \subset \mathbb{R}^{2}$ in its output space. The path $\gamma_{i}, i \in \mathbf{N}$, has a smooth, unit-speed, parameterization $\sigma_{i}: \mathbb{D}_{i} \rightarrow \mathbb{R}^{2}$ with $\gamma_{i}=\sigma_{i}\left(\mathbb{D}_{i}\right)$. If path $i$ is closed then $\mathbb{D}_{i}=\mathbb{R} \bmod L_{i}$ where $L_{i}>0$ is the length of the curve. If path $i$ is non-closed then $\mathbb{D}_{i}=\mathbb{R}$. Let $\varphi_{i}: \mathbb{D}_{i} \rightarrow \mathbb{S}^{1}$ be the map associating to each $\lambda \in \mathbb{D}_{i}$ the angle of the tangent vector $\sigma_{i}^{\prime}(\lambda)$ to $\gamma_{i}$ at $\sigma_{i}(\lambda)$.

Assumption 1. For $i \in \mathbf{N}$, the path $\gamma_{i} \subset \mathbb{R}^{2}$ is a one-dimensional embedded submanifold. There exists a smooth map $s_{i}: \mathbb{R}^{2} \rightarrow \mathbb{R}$ such that $\gamma_{i}=s_{i}^{-1}(0)$ and $\mathrm{d} s_{i}\left(y_{i}\right) \neq 0$ for all $\mathbf{y}_{i} \in \gamma_{i}$. Moreover, there exist $2 N$ class- $\mathcal{K}_{\infty}$ functions $\rho_{i, 1}, \rho_{i, 2}:[0, \infty) \rightarrow \mathbb{R}_{+}$such that for all $\mathbf{y}_{i} \in \mathbb{R}^{2}$

$\rho_{i, 1}\left(\operatorname{dist}\left(\mathbf{y}_{i}, \gamma_{i}\right)\right) \leq\left\|s_{i}\left(\mathbf{y}_{i}\right)\right\| \leq \rho_{i, 1}\left(\operatorname{dist}\left(\mathbf{y}_{i}, \gamma_{i}\right)\right)$.

While the curve $\gamma_{i}$ may be unbounded, condition (2) in Assumption 1 ensures that $\mathbf{y}_{i} \rightarrow \gamma_{i}$ if and only if $s\left(\mathbf{y}_{i}\right) \rightarrow 0$. Our path following objective is to design a smooth feedback controller for each unicycle that drives its closed-loop output towards $\gamma_{i}$. Moreover, we ask that $\gamma_{i}$ be output invariant in the sense defined in Nielsen et al. (2010). Let

$\mathcal{P}_{i}:=\left\{\mathbf{x}_{i} \in \mathbb{R}^{2} \times \mathbb{S}^{1} \times \mathbb{R}: \alpha_{i}\left(\mathbf{x}_{i}\right):=s_{i} \circ h_{i}\left(\mathbf{x}_{i}\right)=0\right\}$

and $\mathcal{P}:=\mathcal{P}_{1} \times \cdots \times \mathcal{P}_{N}$. Driving $\mathbf{x}_{i}$ towards the set $\mathcal{P}_{i}$ corresponds to sending the output $\mathbf{y}_{i}$ of agent $i$ to its desired path. Therefore, we seek to stabilize the largest controlled-invariant subset of $\mathcal{P}_{i}$, denoted $\mathscr{P}_{i}^{\star}$. Intuitively, the set $\mathcal{P}_{i}^{\star}$ is the collection of all those motions of agent $i$ whose associated output signals can be made to lie in $\mathcal{P}_{i}$ at all time by a suitable choice of input signal. The largestcontrolled invariant subset of $\mathcal{P}_{i}$ is (Nielsen \& Maggiore, 2004)

$\mathcal{P}_{i}^{\star}=\left\{\mathbf{x}_{i}: \alpha_{i}\left(\mathbf{x}_{i}\right)=\left\langle\mathrm{d} s_{i}\left(h\left(\mathbf{x}_{i}\right)\right), \tau\left(\theta_{i}\right)\right\rangle=0\right\}$.

This set is called the path following manifold of the unicycle $i$ with respect to $\gamma_{i}$ (Nielsen et al., 2010) and has dimension $n_{i}^{\star}=2$.

Remark 2.5. The set $\mathcal{P}_{i}=\alpha_{i}^{-1}(0)$ is itself controlled invariant because if unicycle $i$ is initialized on $\mathcal{P}_{i}$ with zero translational velocity, $v_{i} \equiv 0$, then $\mathbf{y}_{i}(t) \in \gamma_{i}$ for all $t \geq 0$. This characterization is not useful for coordinated path following because the unicycle cannot traverse its path nor coordinate with other unicycles. This motivates our definition (4). $\triangleleft$

Definition 2.6. The multi-agent path following manifold for $N$ paths $\gamma_{1}, \ldots, \gamma_{N}$ that satisfy Assumption 1, in a neighbourhood of $\mathbf{x}^{\star}=\operatorname{col}\left(\mathbf{x}_{1}^{\star}, \ldots, \mathbf{x}_{\mathrm{N}}^{\star}\right) \in \mathcal{P}$, is

$\mathcal{P}^{\star}:=\mathcal{P}_{1}^{\star} \times \cdots \times \mathcal{P}_{\mathrm{N}}^{\star}$

and its dimension is $n^{\star}=\sum n_{i}^{\star}=2 N$. $\diamond$

\subsection{Coordination}

We model unicycle coordination as a constraint on the allowable motions on the multi-agent path following manifold Definition 2.6.

Definition 2.7. A coordination function on the multi-agent path following manifold is a smooth map $\beta: \mathcal{P}^{\star} \rightarrow \mathbb{R}^{c}, c \leq \operatorname{dim}\left(\mathcal{P}^{\star}\right)$ with constant rank $c$. $\diamond$

Let $\beta: \mathcal{P}^{\star} \rightarrow \mathbb{R}^{c}$ be a coordination function and consider the set

$\mathcal{C}:=\left\{\mathbf{x} \in \mathcal{P}^{\star}: \beta(\mathbf{x})=0\right\}$.
Definition 2.7 and the constant-rank level set theorem (Lee, 2002, Theorem 8.8) imply that the, not necessarily bounded, set $\mathcal{C}$ is a closed embedded submanifold of $\mathcal{P}^{\star}$ of dimension $n^{\star}-c$. We take the view that stabilizing $\mathcal{C}$ corresponds to achieving coordination. This motivates us to characterize the largest controlledinvariant subset of $\mathcal{C}$.

Definition 2.8. Given a point $\mathbf{x} \in \mathcal{C} \subset \mathscr{P}^{\star}$, the local coordination set $\mathcal{C}^{\star}$ associated to a coordination function $\beta: \mathcal{P}^{\star} \rightarrow \mathbb{R}^{c}$ is the largest connected, controlled-invariant subset of $\mathcal{C}$ containing x. $\diamond$

Intuitively, the set $\mathrm{C}^{\star}$ is the collection of all those motions of the multi-agent system whose evolution can be made to satisfy the coordination constraint for all time by a suitable choice of control signal. Communication constraints between unicycles must be respected when characterizing $C^{\star}$. Unless otherwise stated, we make the following simplifying assumption.

Assumption 2. The communication graph $\mathscr{G}$ of the multi-agent unicycle system is complete.

Assumption 2 is unnecessarily restrictive for implementing the proposed control laws. Characterizing the minimal communication requirements needed for implementation is an open problem.

\subsection{Control design objectives}

The coordinated path following control design problem we consider entails finding $N$ feedback control laws ensuring that the closed-loop multi-agent system satisfies:

PF For each initial condition $\mathbf{x}(0)$ in a neighbourhood of $\mathcal{P}^{\star}$, the corresponding solution $\mathbf{x}(t)$ is defined for all $t \geq 0$ and $\mathbf{x}(t) \rightarrow$ $\mathcal{P}^{\star}$ as $t \rightarrow+\infty$.

C1 For each initial condition $\mathbf{x}(0)$ in a neighbourhood of $\mathcal{C}^{\star}$, with $\mathbf{x}(0) \in \mathscr{P}^{\star}$, the corresponding solution $\mathbf{x}(t) \in \mathcal{P}^{\star}$ for all $t \geq 0$ and $\mathbf{x}(t) \rightarrow \mathcal{C}^{\star}$.

C2 The dynamics of the multi-agent system restricted to $\mathcal{C}^{\star}$ satisfy application specific specifications, e.g., boundedness, tracking, etc.

C3 The proposed control laws respect the communication constraints imposed by the communication graph $\mathscr{G}$.

While PF can be solved locally by each unicycle, C1 and C2 generally require the unicycles to exchange information. Under Assumption 2, C3 is trivially satisfied. We loosen Assumption 2 in Section 5.4

We have cast the coordinated path following problem as two set stabilization problems; namely the stabilization of $\mathcal{P}^{\star}$ and $\mathcal{C}^{\star}$. In this study we first stabilize $\mathcal{P}^{\star}$ and then $\mathcal{C}^{\star}$. While one could, in principle, achieve coordinated path following problem by directly stabilizing $\mathcal{C}^{\star}$, we take a nested set stabilization approach. This approach has two distinct advantages. First, it ensures that even if coordination fails due to, say, communication errors, the individual unicycles remain on their paths. Second, it allows one to change the coordination specification without causing the robots to leave their paths.

Remark 2.9. For general multi-agent systems, even if the closedloop system satisfies PF and C1, there is no guarantee that when $\mathbf{x}(0) \notin \mathcal{P}^{\star}$ that $\mathbf{x}(t) \rightarrow \mathcal{C}^{\star}$. For unicycles, the results of Section 5.2 prove that this problem does not occur. $\triangleleft$

\section{The multi-agent path following manifold}

\subsection{Characterization of the multi-agent path following manifold}

By Definition 2.6, the multi-agent path following manifold is the product of each agent's individual path following manifold.

Proposition 3.1. For $i \in \mathbf{N}$ the set $\mathcal{P}_{i}^{\star} \backslash\left\{v_{i}=0\right\}$, where $\mathcal{P}_{i}^{\star}$ is given by (4), consists of four disconnected components. 


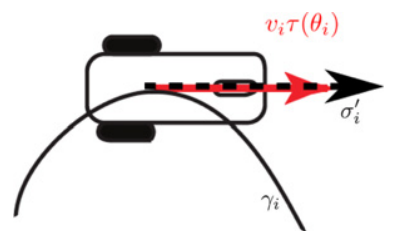

(a) $\mathcal{P}_{i,+}^{\star, \mathrm{f}}$.

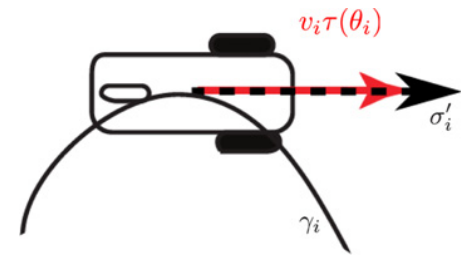

(b) $\mathcal{P}_{i,-}^{\star, \mathrm{r}}$.
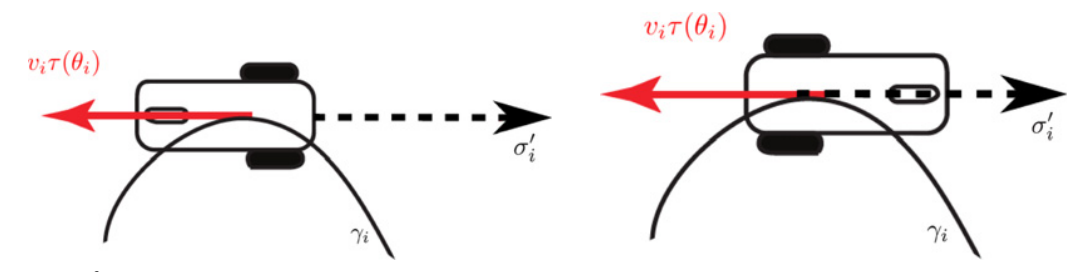

(c) $\mathcal{P}_{i,-}^{\star,}$.

(d) $\mathcal{P}_{i,+}^{\star, r}$.

Fig. 1. The motion of unicycle $i$ restricted to the four components of $\mathcal{P}_{i}^{\star} \backslash\left\{v_{i}=0\right\}$.

Proof. Fix $i \in \mathbf{N}$ and consider the set $\mathcal{P}_{i, \bullet}^{\star, \bullet}:=\mathcal{P}_{i,+}^{\star, \mathrm{f}} \cup \mathcal{P}_{i,+}^{\star, \mathrm{r}} \cup \mathcal{P}_{i,-}^{\star, \mathrm{f}} \cup$ $\mathcal{P}_{i,-}^{\star, \mathrm{r}}$ where

$\mathcal{P}_{i,+}^{\star, \mathrm{f}}:=\left\{\mathbf{x}_{i} \in \mathcal{P}_{i}^{\star}: v_{i}>0,\left\langle\sigma_{i}^{\prime}(\lambda), \tau\left(\theta_{i}\right)\right\rangle=1, \lambda \in \mathbb{D}_{i}\right\}$

$\mathcal{P}_{i,+}^{\star, \mathrm{r}}:=\left\{\mathbf{x}_{i} \in \mathcal{P}_{i}^{\star}: v_{i}<0,\left\langle\sigma_{i}^{\prime}(\lambda), \tau\left(\theta_{i}\right)\right\rangle=1, \lambda \in \mathbb{D}_{i}\right\}$

$\mathcal{P}_{i,-}^{\star, \mathrm{f}}:=\left\{\mathbf{x}_{i} \in \mathcal{P}_{i}^{\star}: v_{i}>0,\left\langle\sigma_{i}^{\prime}(\lambda), \tau\left(\theta_{i}\right)\right\rangle=-1, \lambda \in \mathbb{D}_{i}\right\}$

$\mathcal{P}_{i,-}^{\star, \mathrm{r}}:=\left\{\mathbf{x}_{i} \in \mathcal{P}_{i}^{\star}: v_{i}<0,\left\langle\sigma_{i}^{\prime}(\lambda), \tau\left(\theta_{i}\right)\right\rangle=-1, \lambda \in \mathbb{D}_{i}\right\}$.

We first show that $\mathcal{P}_{i, \bullet}^{\star \bullet}$ has four disconnected components, namely the sets (7). The sets $\mathcal{P}_{i,+}^{\star, \mathrm{f}} \cup \mathcal{P}_{i,-}^{\star, \mathrm{f}}$ and $\mathcal{P}_{i,+}^{\star, \mathrm{r}} \cup \mathcal{P}_{i,-}^{\star, \mathrm{r}}$ are disjoint because any curve in the state space connecting these sets must pass through a point at which $v_{i}=0$.

Next assume, without loss of generality, that $\overline{\mathbf{x}}_{i} \in \mathcal{P}_{i,+}^{\star, \mathrm{f}}$. To connect $\overline{\mathbf{x}}_{i}$ to another point $\overline{\overline{\mathbf{x}}}_{i} \in \mathcal{P}_{i,-}^{\star, \mathrm{f}}$ it has to pass through a point corresponding to $\left\langle\sigma_{i}^{\prime}(\lambda), \tau\left(\theta_{i}\right)\right\rangle=0$. This shows that $\mathcal{P}_{i,+}^{\star, \mathrm{f}}$ and $\mathcal{P}_{i,-}^{\star, \mathrm{f}}$ are not path connected. A similar argument holds for $\mathcal{P}_{i,+}^{\star, \mathrm{r}}$ and $P_{i,-}^{\star, \mathrm{r}}$. Together, these facts show that $\mathcal{P}_{i, \bullet}^{\star} \bullet$ is not path connected which implies that, see Lee (2002, Proposition 1.8), it is not connected.

Lastly, we show that $\mathcal{P}_{i, \bullet}^{\star, \bullet}=\mathcal{P}_{i}^{\star} \backslash\left\{v_{i}=0\right\}$. By definition we have that $\mathcal{P}_{i, \bullet}^{\star} \subseteq \mathcal{P}_{i}^{\star}$. Conversely, let $\mathbf{x}_{i}=\left(x_{i}, y_{i}, \theta_{i}, v_{i}\right) \in \mathcal{P}_{i}^{\star}$ with $v_{i} \neq 0$. Since $\mathcal{P}_{i}^{\star}$ is an invariant set contained in $\mathcal{P}_{i}$, the unicycle's heading must be tangent to the path for, otherwise, it would leave the path for some time and hence leave the set $\mathcal{P}_{i}$. This implies that $\left|\left\langle\sigma_{i}^{\prime}(\lambda), \tau\left(\theta_{i}\right)\right\rangle\right|=1$ where $\lambda \in \mathbb{D}_{i}$ satisfies $\mathbf{y}_{i}=\sigma_{i}(\lambda)$. This shows that $\mathcal{P}_{i}^{\star} \subseteq \mathcal{P}_{i, \bullet}^{\star} \bullet$

The notation in (7) is evocative of the physical interpretation of these four sets, see Fig. 1. The superscript $f$ stands for forward direction, the superscript $r$ stands for reverse direction, the subscript + indicates the unicycle is moving in the same direction as curve's orientation, and subscript - indicates the unicycle is moving opposite to the curve's orientation.

\subsection{Unicycle normal form}

Using local transverse feedback linearization (Nielsen \& Maggiore, 2008) we transform the model of unicycle $i$ to a convenient normal form. Among other useful properties, the normal form suggests local coordinates on $\mathcal{P}_{i}^{\star}$ that simplify finding the coordination set. It also facilitates the design of decentralized control laws to stabilize $\mathcal{P}^{\star}$.

Inspired by Consolini, Maggiore, Nielsen, and Tosques (2010), we introduce a projection in the output space of the unicycle that associates to each point $\mathbf{y}_{i}$ sufficiently close to the path $\gamma_{i}$ a number in $\mathbb{D}_{i}$. Let

$$
\begin{aligned}
\varpi_{i}: \mathcal{N}\left(\gamma_{i}\right) & \rightarrow \mathbb{D}_{i} \\
\mathbf{y}_{i} & \mapsto \arg \inf _{\lambda \in \mathbb{D}_{i}}\left\|\mathbf{y}_{i}-\sigma_{i}(\lambda)\right\|
\end{aligned}
$$

where $\mathcal{N}\left(\gamma_{i}\right)$ is a neighbourhood of $\gamma_{i}$. The open set $\mathcal{N}\left(\gamma_{i}\right)$ is such that, for all $\mathbf{y}_{i} \in \mathcal{N}\left(\gamma_{i}\right)$, there exists a unique $\mathbf{y}_{i}^{\star} \in \gamma_{i}$ closest to $\mathbf{y}_{i}$, so $\varpi_{i}$ is well-defined. The higher the curvature of the path $\gamma_{i}$, the smaller the domain $\mathcal{N}\left(\gamma_{i}\right)$ of (8). Using (8) define $\pi_{i}\left(\mathbf{x}_{i}\right):=$ $\varpi_{i} \circ h_{i}\left(\mathbf{x}_{i}\right)$. Finally, define the path following output function

$\hat{\mathbf{y}}_{i}:=\left(\pi_{i}\left(\mathbf{x}_{i}\right), \alpha_{i}\left(\mathbf{x}_{i}\right)\right)$.

Lemma 3.2. The unicycle (1) with output (9) yields a well-defined vector relative degree of $\{2,2\}$ at each $\mathbf{x}_{i} \in \mathcal{P}_{i}^{\star} \backslash\left\{v_{i}=0\right\}$.

The proof of Lemma 3.2 is omitted because it is similar to Akhtar, Nielsen, and Waslander (2015, Lemma 3.1). The next lemma defines a coordinate transformation valid in a neighbourhood of each component (7) of $\mathcal{P}_{i}^{\star} \backslash\left\{v_{i}=0\right\}$. The lemma explicitly addresses the component $\mathcal{P}_{i,+}^{\star, f}$ but a similar result can be obtained for the remaining three components of $\mathcal{P}_{i}^{\star}$.

Lemma 3.3. There exists an open set $U_{i,+}^{\mathrm{f}} \subseteq \mathbb{R}^{2} \times \mathbb{S}^{1} \times \mathbb{R}$, with $\mathcal{P}_{i,+}^{\star, \mathrm{f}} \subset$ $U_{i,+}^{\mathrm{f}}$ such that $T_{i}: U_{i,+}^{\mathrm{f}} \rightarrow T_{i}\left(U_{i,+}^{\mathrm{f}}\right), \mathbf{x}_{i} \mapsto\left(\eta_{i, 1}, \eta_{i, 2}, \xi_{i, 1}, \xi_{i, 2}\right)=$ $\left(\pi_{i}\left(\mathbf{x}_{i}\right), L_{f} \pi_{i}\left(\mathbf{x}_{i}\right), \alpha_{i}\left(\mathbf{x}_{i}\right), L_{f} \alpha_{i}\left(\mathbf{x}_{i}\right)\right)$ is a diffeomorphism onto its image.

Proof. The generalized inverse function theorem (Guillemin \& Pollack, 2010, p. 56) is employed to prove this result. We must show that

(1) for all $\mathbf{x}_{i} \in \mathcal{P}_{i+}^{\star, \mathrm{f}}, \mathrm{d} T_{i}\left(\mathbf{x}_{i}\right)$ is an isomorphism

(2) $\left.T_{i}\right|_{\mathcal{P}_{i,+}^{\star, f}}$ is a diffeomorphism.

To show that (1) holds, observe that $\left.\operatorname{det}\left(\mathrm{d} T_{i}\right)\right|_{\mathcal{P}_{i,+}^{\star, f}}=-v_{i}\left(\partial_{x_{i}} \pi_{i} \partial_{y_{i}} \alpha_{i}\right.$ $\left.-\partial_{x_{i}} \alpha_{i} \partial_{y_{i}} \pi_{i}\right)^{2}$. On $\mathcal{P}_{i,+}^{\star, \mathrm{f}}, v_{i} \neq 0$. Using arguments analogous to those in Consolini et al. (2010, Lemma 3.2), $\mathrm{d} \alpha_{i}=\left[\begin{array}{llll}\partial_{x_{i}} \alpha_{i} & \partial_{y_{i}} \alpha_{i} & 0 & 0\end{array}\right]$ and $\mathrm{d} \pi_{i}=\left[\begin{array}{llll}\partial_{x_{i}} \pi_{i} & \partial_{y_{i}} \pi_{i} & 0 & 0\end{array}\right]$ are orthogonal on $\mathcal{P}_{i,+}^{\star, \mathrm{f}}$ and so $\left(\partial_{x_{i}} \pi_{i} \partial_{y_{i}} \alpha_{i}-\partial_{x_{i}} \alpha_{i} \partial_{y_{i}} \pi_{i}\right) \neq 0$.

To show that (2) holds, note that the restriction of $T_{i}$ to $\mathcal{P}_{i,+}^{\star, \mathrm{f}}$ is given by $\left(\eta_{i, 1}, \eta_{i, 2}, \xi_{i, 1}, \xi_{i, 2}\right)=\left(\pi_{i}\left(\mathbf{x}_{i}\right), L_{f} \pi_{i}\left(\mathbf{x}_{i}\right), 0,0\right)$. For $\mathbf{x}_{i} \in \mathcal{P}_{i,+}^{\star, \mathrm{f}}$ we have that $\mathbf{y}_{i}=h\left(\mathbf{x}_{i}\right) \in \gamma_{i}$ and so by the definition of $\eta_{i, 1}=$ $\pi_{i}\left(\mathbf{x}_{i}\right),\left(x_{i}, y_{i}\right)=\sigma_{i}\left(\eta_{i, 1}\right)$. The vector $\sigma^{\prime}\left(\eta_{i, 1}\right)$ is tangent to the curve $\gamma_{i}$ at $\sigma_{i}\left(\eta_{i, 1}\right)$. On the component $\mathcal{P}_{i,+}^{\star, \mathrm{f}}$ of $\mathcal{P}_{i}^{\star}, \tau\left(\theta_{i}\right)=\sigma^{\prime}\left(\eta_{i, 1}\right)$ and therefore $\theta_{i}=\varphi_{i}\left(\eta_{i, 1}\right)$. 
We are left to find an expression for $v_{i}$. Direct calculations using the expression $\eta_{i, 2}=L_{f} \pi_{i}\left(\mathbf{x}_{i}\right)$ yield $v_{i}=\eta_{i, 2} /\left(\partial_{x_{i}} \pi_{i} \cos \theta_{i}+\right.$ $\partial_{y_{i}} \pi_{i} \sin \theta_{i}$ ).

By Consolini et al. (2010, Lemma 3.2), for all $\eta_{i, 1} \in \mathbb{D}_{i}$, $\mathrm{d} \varpi_{i}\left(\sigma_{i}\right.$ $\left.\left(\eta_{i, 1}\right)\right)=\sigma_{i}^{\prime}\left(\eta_{i, 1}\right)$. Therefore, for $\mathbf{x}_{i} \in \mathcal{P}_{i,+}^{\star, \mathrm{f}}, \mathrm{d} \pi_{i}\left(\mathbf{x}_{i}\right)=\left[\sigma_{i}^{\prime}\left(\eta_{i, 1}\right)^{\top}\right.$

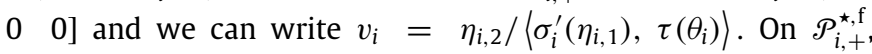
$\left\langle\sigma^{\prime}\left(\eta_{i, 1}\right), \tau\left(\theta_{i}\right)\right\rangle=1$ so $v_{i}=\eta_{i, 2}$. In summary, we have derived the inverse map $\left.T_{i}^{-1}\right|_{\mathcal{P}_{i,+}^{\star, f}}=\left(\sigma_{i}\left(\eta_{i, 1}\right), \varphi_{i}\left(\eta_{i, 1}\right), \eta_{i, 2}\right)$ which shows that $\left.T_{i}\right|_{\mathcal{P}_{i,+}^{\star, f}}$ is a diffeomorphism onto its image.

By Lemma 3.2 the regular feedback transformation

$$
\left[\begin{array}{l}
u_{i, 1} \\
u_{i, 2}
\end{array}\right]=\frac{1}{v_{i}^{2}}\left[\begin{array}{cc}
L_{g_{i, 2}} L_{f} \alpha_{i} & -L_{g_{i, 2}} L_{f} \pi_{i} \\
-L_{g_{i, 1}} L_{f} \alpha_{i} & L_{g_{i, 1}} L_{f} \pi_{i}
\end{array}\right]\left[\begin{array}{c}
-L_{f}^{2} \alpha+v_{i}^{\|} \\
-L_{f}^{2} \pi+v_{i}^{\pitchfork}
\end{array}\right],
$$

where $\left(v_{i}^{\|}, v_{i}^{\pitchfork}\right) \in \mathbb{R} \times \mathbb{R}$ are auxiliary control inputs, is well defined in a neighbourhood of $\mathcal{P}_{i}^{\star} \backslash\left\{v_{i}=0\right\}$. The elements in (10) can be readily computed (Akhtar et al., 2015, Section V). Using the diffeomorphism $T_{i}$ in Lemma 3.3, and the feedback transformation (10), the dynamic unicycle (1) is feedback equivalent, in a neighbourhood of each component of $\mathcal{P}_{i}^{\star} \backslash\left\{v_{i}=0\right\}$, to

$$
\begin{aligned}
& \dot{\eta}_{i, 1}=\eta_{i, 2} \\
& \dot{\eta}_{i, 2}=v_{i}^{\|} \\
& \dot{\xi}_{i, 1}=\xi_{i, 2} \\
& \dot{\xi}_{i, 2}=v_{i}^{\dagger} .
\end{aligned}
$$

Remark 3.4. We stress that unicycle $i$ is not globally feedback equivalent to (11). Furthermore, the equivalence does not hold when its translational velocity equals zero $v_{i}=0$. The latter obstacle can be overcome using the switching scheme in Tomlin and Sastry (1998). $\triangleleft$

We call the subsystem (11b) in (11) the transversal dynamics of unicycle $i$ to each component of $\mathcal{P}_{i}^{\star}$. This is because making each component of $\mathcal{P}_{i}^{\star}$ attractive is equivalent, under Assumption 1 and in particular Eq. (2), to stabilizing the origin of (11b). The subsystem (11a) is called tangential dynamics of unicycle $i$ with respect to each component of $\mathcal{P}_{i}^{\star}$. The $\eta_{i, 1}$ and $\eta_{i, 2}$ states convey a strong physical meaning for coordinated path following. The state $\eta_{i, 1}$ represents the position of unicycle $i$ along the path and the state $\eta_{i, 2} \in \mathbb{R}$ represents its velocity along the path. Let $\boldsymbol{\xi}:=$ $\left(\xi_{1,1}, \ldots, \xi_{N, 1}, \xi_{1,2}, \ldots, \xi_{N, 2}\right)$ denote the transversal states of the entire multi-agent unicycle system and $\mathbf{v}^{\dagger}:=\left(v_{1}^{\pitchfork}, \ldots, v_{N}^{\pitchfork}\right)$. Let $\boldsymbol{\eta}_{1}:=\left(\eta_{1,1}, \ldots, \eta_{N, 1}\right), \boldsymbol{\eta}_{2}:=\left(\eta_{1,2}, \ldots, \eta_{N, 2}\right), \boldsymbol{\eta}:=\left(\boldsymbol{\eta}_{1}, \boldsymbol{\eta}_{2}\right)$, and $\mathbf{v}^{\|}:=\left(v_{1}^{\|}, \ldots, v_{N}^{\|}\right)$then the overall dynamics of the multi-agent system can be compactly written as

$$
\begin{aligned}
& \dot{\boldsymbol{\eta}}=A^{\|} \boldsymbol{\eta}+B^{\|} \mathbf{v}^{\|} \\
& \dot{\boldsymbol{\xi}}=A^{\dagger} \boldsymbol{\xi}+B^{\dagger} \mathbf{v}^{\dagger}
\end{aligned}
$$

where $\left(A^{\pitchfork}, B^{\pitchfork}\right),\left(A^{\|}, B^{\|}\right)$are controllable. The dynamics of the multi-agent system restricted to $\mathcal{P}^{\star}$ are given by (12a) and these dynamics play a key role in achieving coordination.

\subsection{Topology of multi-agent path following manifold}

The tangential dynamics (11a) evolve on the set (4). When the curve $\gamma_{i}$ is non-closed then $\mathbb{D}_{i}=\mathbb{R}$ and the path is unbounded. In this case $\eta_{i, 1} \in \mathbb{R}$ and $\eta_{i, 2} \in \mathbb{R}$; thus each component of $\mathcal{P}_{i}^{\star} \backslash\left\{v_{i}=0\right\}$ is diffeomorphic to $\mathbb{R} \times \mathbb{R}$. When the path $\gamma_{i}$ is closed then, by Assumption 1, it is a Jordan curve. In this case $\eta_{i, 1} \in$ $\mathbb{R} \bmod L_{i} \simeq \mathbb{S}^{1}$ and $\eta_{i, 2} \in \mathbb{R}$; thus each component of $\mathcal{P}_{i}^{\star} \backslash\left\{v_{i}=0\right\}$ is diffeomorphic to $\mathbb{S}^{1} \times \mathbb{R}$.
Assume, without loss of generality, that $\gamma_{i}$ is closed for $i \in \mathbf{r}$, $r \leq N$ and non-closed for $i \in\{r+1, \ldots, N\}$. Then each component of the multi-agent path following manifold is diffeomorphic to $\mathbb{T}^{r} \times \mathbb{R}^{N-r} \times \mathbb{R}^{N}$ where $\mathbb{T}^{r}$ is the $r$-torus. This shows that $\mathcal{P}^{\star}$ is unbounded even if every curve is closed.

As shown in the proof of Lemma 3.3, the tangential states in (11a) represent local coordinates on each component of $\mathcal{P}_{i}^{\star} \backslash\left\{v_{i}=0\right\}$. When $\gamma_{i} \simeq \mathbb{R}$ then $\left(\mathcal{P}_{i,+}^{\star, f}, \psi_{i}\right)$ with $\psi_{i}:=\left(\left.\pi_{i}\right|_{\mathcal{P}_{i}^{\star}}\right.$, $\left.\left.L_{f} \pi_{i}\right|_{\mathcal{P}_{i}^{\star}}\right)$ is a global coordinate chart, i.e., a single chart that covers the entire set $\mathcal{P}_{i,+}^{\star, \mathrm{f}}$. When $\gamma_{i}$ is closed each component of $\mathcal{P}_{i}^{\star} \backslash\left\{v_{i}=0\right\}$ is diffeomorphic to $\mathbb{S}^{1} \times \mathbb{R}$ and cannot be covered with a single chart. Instead the coordinate chart $\left(U_{i}, \psi_{i}\right)$ with $U_{i}:=$ $\left(\mathbb{R} \bmod L_{i}\right) \backslash\{0\} \times \mathbb{R} \subset \mathcal{P}_{i,+}^{\star, \mathrm{f}}$ covers "most" of $\mathcal{P}_{i,+}^{\star, \mathrm{f}}$. One could define another chart to cover the omitted region, but this complication is not needed (Bullo \& Lewis, 2005). Since $\mathcal{P}^{\star}$ is a product manifold, $(U, \psi)$ with $U:=U_{1} \times \cdots \times U_{N}$ and $\psi:=\psi_{1} \times \cdots \times \psi_{N}$ is a coordinate chart for $\mathcal{P}^{\star}$. When all the paths are non-closed it covers the entire set $\mathcal{P}^{\star}$, otherwise it covers "most" of $\mathcal{P}^{\star}$.

\section{Feasible coordination constraints}

Let $\beta: \mathcal{P}^{\star} \rightarrow \mathbb{R}^{c}$ be a coordination function, see Definition 2.7. As discussed in Section 3.3 each component of $\mathcal{P}^{\star}$ is diffeomorphic to $\mathbb{T}^{r} \times \mathbb{R}^{N-r} \times \mathbb{R}^{N}$ and the coordinate chart $(U, \psi)$ covers most of it. In this section we work with a local representation of the coordination function, $\hat{\beta}: \psi(U) \rightarrow \mathbb{R}^{c}$, defined by

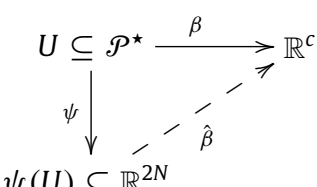

Remark 4.1. In order to define a coordination function globally, and avoid the use of charts on $\mathcal{P}^{\star}$, one uniquely identifies smooth functions on the $r$-torus with smooth periodic functions on $\mathbb{R}^{r}$. In light of the discussion in Section 3.3 we can then treat $\hat{\beta}$ as a map $\mathbb{R}^{2 N} \rightarrow \mathbb{R}^{c}, L_{i}$-periodic in its first $r$ arguments (Bhat \& Bernstein, 2000). $\triangleleft$

For $\eta \in \psi(U)$ the associated local coordination set introduced in Definition 2.8 is the largest controlled-invariant subset of

$\mathcal{C}=\{\boldsymbol{\eta} \in \psi(U): \hat{\beta}(\boldsymbol{\eta})=0\}$

containing $\eta$. A coordination specification is feasible if its corresponding coordination set is non-empty.

\subsection{Linear-affine coordination}

Consider a linear-affine coordination function

$\mathcal{C}=\{\eta \in \psi(U): \hat{\beta}(\eta)=A \eta+b=0\}$

with $A \in \mathbb{R}^{c \times 2 N}, \operatorname{rank}(A)=c$, and $b \in \operatorname{Im} A$. We write $A=$ $\left[\begin{array}{ll}A_{1} & A_{2}\end{array}\right]$ and $b=\left(b_{1}, b_{2}\right)$ in accordance with the partition $\boldsymbol{\eta}=$ $\left(\eta_{1}, \eta_{2}\right)$.

Proposition 4.2. Consider the set (14) and let $R$ be a full rank matrix satisfying $R A_{2}=0$. If

(1) $\left(\forall \eta_{2} \in \mathcal{C}\right) R A_{1} \eta_{2}=0$, or

(2)

$$
\operatorname{Im}\left[\begin{array}{l}
b \\
0
\end{array}\right] \in \operatorname{Im}\left[\begin{array}{cc}
A_{1} & A_{2} \\
0 & R A_{1}
\end{array}\right]
$$

then, for each $\eta \in \mathcal{C}$, the set $\mathcal{C}^{\star}$ is non-empty. 
Proof. By the definition of (14), $b \in \operatorname{Im} A$, therefore the set $\mathcal{C}$ is non-empty. For the set $C$ itself to be controlled invariant there must exist a control law $\mathbf{v}^{\|}$such that the derivative of $A \eta+b$ is identically zero. Taking the derivative of $\hat{\beta}$ along solutions of the system (12a) we obtain

$A_{1} \eta_{2}+\left.A_{2} \mathbf{v}^{\|}\right|_{c}=0$.

Left multiply Eq. (16) by $R$ to obtain that Eq. (16) is solvable in $\mathbf{v}^{\|}$ if and only if $\left.R A_{1} \eta_{2}\right|_{\mathcal{C}}=0$. In this case $\mathcal{C}^{\star}=\mathcal{C}$ and is non-empty. If, on the other hand, there exists $\eta_{2} \in \mathcal{C}$ for which $R A_{1} \eta_{2} \neq 0$, then (16) is not solvable using $\mathbf{v}^{\|}$. In this case we add the constraint $R A_{1} \eta_{2}=0$ to $\mathcal{C}$ and obtain a new set $\mathcal{C}_{1}=\left\{\eta \in \mathcal{C}: R A_{1} \eta_{2}=0\right\}$. In order for the set $\mathcal{C}_{1}$ to be controlled invariant there must exist a control law $\mathbf{v}^{\|}$such that the derivative of $R A_{1} \eta_{2}$ along solutions of system (12a) is identically zero. Setting the derivative equal to zero we obtain $\left.R A_{1} \mathbf{v}^{\|}\right|_{c_{1}}=0$. Any feedback control law that satisfies, for all $\eta \in \mathcal{C}_{1}, \mathbf{v}^{\|}(\boldsymbol{\eta}) \in \operatorname{Ker}\left(R A_{1}\right)$, solves this equation, so the coordination set, $\mathcal{C}^{\star}$, equals $\mathcal{C}_{1}$. Condition (15) guarantees that $\mathcal{C}_{1}$ is non-empty.

\subsubsection{Nonlinear coordination}

Consider a coordination specification described by a nonlinear coordination function $\hat{\beta}: \psi(U) \subseteq \mathbb{R}^{2 N} \rightarrow \mathbb{R}^{c}$. We emphasize that either (i) $\hat{\beta}$ is the local representation of a coordination function in a chart $(U, \psi)$ or (ii) it is a global function $\hat{\beta}: \mathbb{R}^{2 N} \rightarrow \mathbb{R}^{c}$ which is $L_{i}$-periodic in its first $r$ arguments. By Definition 2.7 the set $(13)$ is a smooth $(2 N-c)$-dimensional embedded submanifold of $\psi(U)$. Consider the partition for $\mathrm{d} \hat{\beta}(\boldsymbol{\eta})=\left[\begin{array}{lll}\partial_{\eta_{1}} \hat{\beta} & \partial_{\eta_{2}} \hat{\beta}\end{array}\right]$ in accordance with $\boldsymbol{\eta}=\left(\boldsymbol{\eta}_{1}, \boldsymbol{\eta}_{2}\right)$. Propositions 4.3 and 4.4 give sufficient conditions for velocity coordination and position coordination constraints to be feasible.

Proposition 4.3 (Nonlinear Velocity Coordination). If the coordination function satisfies $\partial_{\eta_{1}} \hat{\beta} \equiv 0$ then, for each $\eta \in \mathcal{C}, \mathcal{C}^{\star}=\mathcal{C}$ and is non-empty.

Proof. To check whether or not $\mathcal{C}$ is controlled-invariant we take the derivative of $\hat{\beta}$ along solutions of the system (12a) to obtain $\partial_{\eta_{2}} \hat{\beta} \mathbf{v}^{\|}=0$. Since $\mathbf{v}^{\|}=0$ trivially solves this equation we have $\mathcal{C}^{\star}=\mathcal{C}$.

Proposition 4.4 (Nonlinear Position Coordination). If the coordination function satisfies $\partial_{\eta_{2}} \hat{\beta} \equiv 0$ and $\eta \in \mathcal{C}$ is such that $\partial_{\eta_{1}}\left(\partial_{\eta_{1}}\right.$ $\left.\hat{\beta} \eta_{2}\right) \eta_{2} \in \operatorname{Im} \partial_{\eta_{1}} \hat{\beta}$ then, the local coordination set $\mathcal{C}^{\star}$ is non-empty.

Proof. By assumption, the derivative of $\hat{\beta}$ along solutions of (12a) equals $\partial_{\eta_{1}} \hat{\beta} \eta_{2}=0$. Since no control inputs appear we impose the additional constraint $\partial_{\eta_{1}} \hat{\beta} \eta_{2}=0$ to the set $\mathcal{C}$ and obtain $\mathcal{C}_{1}=$ $\left\{\boldsymbol{\eta} \in \mathcal{C}: \partial_{\eta_{1}} \hat{\beta} \eta_{2}=0\right\}$. This set is a closed-embedded submanifold of dimension $2 N-2 c \geq 0$ because its defining constraints are a submersion

$\operatorname{rank}\left[\begin{array}{cc}\partial_{\eta_{1}} \hat{\beta} & \mathbf{0}_{N} \\ \partial_{\eta_{1}}\left(\partial_{\eta_{1}} \hat{\beta} \eta_{2}\right) & \partial_{\eta_{1}} \hat{\beta}\end{array}\right]=2 c \leq 2 N$.

In order to check controlled-invariance of $\mathcal{C}_{1}$ we take the derivative of the constraint $\partial_{\eta_{1}} \hat{\beta} \boldsymbol{\eta}_{2}=0$ along solutions of (12a) to obtain $\partial_{\eta_{1}}\left(\partial_{\eta_{1}} \hat{\beta} \boldsymbol{\eta}_{2}\right) \boldsymbol{\eta}_{2}+\partial_{\eta_{1}} \hat{\beta} \mathbf{v}^{\|}=0$. The condition $\partial_{\eta_{1}} \hat{\beta}\left(\partial_{\eta_{1}} \hat{\beta} \boldsymbol{\eta}_{2}\right) \boldsymbol{\eta}_{2} \in$ $\operatorname{Im} \partial_{\eta_{1}} \beta$ guarantees that the above equation is solvable in $\mathbf{v}^{\|}$for $\eta \in \mathcal{C}_{1} \subseteq \mathcal{C}$ and therefore $\mathcal{C}^{\star}=\mathcal{C}_{1}$.

\section{Control design}

We now design feedback controllers to solve our coordinated path following problem.

\subsection{Stabilizing the multi-agent path following manifold}

To accomplish PF the multi-agent path following manifold $\mathcal{P}^{\star}$ must be made attractive and controlled invariant. As discussed in Section 3.2, this can be done by asking each unicycle to stabilize its own component $\mathcal{P}_{i}^{\star}$. To stabilize $\mathcal{P}_{i}^{\star}$ we select the simplest transversal controller for unicycle $i$

$v_{i}^{\pitchfork}\left(\xi_{i}\right)=-k_{i, 1} \xi_{i, 1}-k_{i, 2} \xi_{i, 2}$

with $k_{i, 1}, k_{i, 2}>0, i \in \mathbf{N}$. For fast convergence to the path one typically chooses the gains so that the roots of the polynomial $s^{2}+k_{i, 2} s+k_{i, 1}$ are far to left in the open left-half complex plane. Alternatively, optimal linear quadratic regulation or model predictive control can be employed when actuator constraints are a concern. With the above choice the origin of each transversal subsystem is rendered exponentially stable.

Remark 5.1. If the trajectory of the unicycle is bounded, then stabilizing $\left(\xi_{i, 1}, \xi_{i, 2}\right)=0$ is equivalent to stabilizing $\mathcal{P}_{i}^{\star}$. When $\mathbb{D}_{i}=\mathbb{R}$ the path $\gamma_{i}$ itself is unbounded and so traversing the path results in unbounded trajectories for the unicycle. In that case, Assumption 1 and in particular (2) ensures that $\left(\xi_{i, 1}, \xi_{i, 2}\right) \rightarrow$ $0 \Longleftrightarrow \mathbf{x}_{i} \rightarrow \mathcal{P}_{i}^{\star}$ (El-Hawwary \& Maggiore, 2010). The component of $\mathcal{P}_{i}^{\star}$ that the unicycle approaches depends on initial conditions. $\triangleleft$

\subsection{Centralized stabilization of the coordination set}

Given a feasible local coordination set $\mathcal{C}^{\star} \subseteq \psi(U)$ and $\eta \in \mathcal{C}^{\star}$ we seek to, under Assumption 2, feedback linearize that portion of the tangential dynamics (12a) that governs whether or not coordination is being achieved. This is equivalent to the following zero dynamics assignment problem (Nielsen \& Maggiore, 2008): Find a function $\tilde{\beta}: V \subseteq \psi(U) \rightarrow \mathbb{R}^{c}, V$ is an open set containing $\eta$ such that (i) $\tilde{\beta}$ yields a well-defined vector relative degree for the tangential dynamics (12a) at $\boldsymbol{\eta}$ and (ii) the associated zero dynamics manifold equals $\mathcal{C}^{\star} \cap V$. Necessary and sufficient conditions to determine the existence of such a function are given in Nielsen and Maggiore (2008). If such a function exists then, in a neighbourhood of $\boldsymbol{\eta}$, the tangential dynamics (12a) are locally feedback equivalent to

$\dot{\zeta}=f(\zeta, \mu)+g^{\pitchfork}(\zeta, \mu) \tau^{\pitchfork}+g^{\|}(\zeta, \mu) \tau^{\|}$

$\dot{\mu}=A \boldsymbol{\mu}+B \boldsymbol{\tau}^{\pitchfork}$

where $(\boldsymbol{\zeta}, \boldsymbol{\mu}) \in \mathbb{R}^{c^{\star}} \times \mathbb{R}^{2 N-c^{\star}}, c^{\star}:=\operatorname{dim} \mathcal{C}^{\star},(A, B)$ controllable, and $\mathcal{C}^{\star}$, expressed in $(\boldsymbol{\zeta}, \boldsymbol{\mu})$-coordinates, is given by $\{(\boldsymbol{\zeta}, \boldsymbol{\mu}): \boldsymbol{\mu}=0\}$. A natural candidate for the function $\tilde{\beta}$ is the coordination function $\hat{\beta}$ itself. We explore this possibility in Propositions 5.5 and 5.6.

In (18) the $\boldsymbol{\mu}$-subsystem describes the motion transversal to the set $\mathcal{C}^{\star}$ but tangential to $\mathcal{P}^{\star}$. Since $(A, B)$ is controllable, there exists a linear feedback $\boldsymbol{\tau}^{\hbar}=F \boldsymbol{\mu}$ that exponentially stabilizes the origin of the $\boldsymbol{\mu}$-subsystem. Then, because the set $\mathcal{C}^{\star}$ is not necessarily bounded, under similar caveats as those discussed in Remark 5.1 the set $\mathcal{C}^{\star}$ is rendered locally attractive and invariant and specification $\mathbf{C 1}$ is achieved.

The $\zeta$-subsystem in (18) describes the dynamics tangent to both $\mathcal{C}^{\star}$ and $\psi(U)$. When restricted to $\mathcal{C}^{\star}$, the multi-agent unicycle system evolves according to

$\dot{\zeta}=f(\zeta, 0)+g^{\|}(\zeta, 0) \tau^{\|}$.

System (19) models the group dynamics while restricted to evolve on the assigned paths and restricted to coordinated motion. In some cases it may be possible to use the remaining control inputs $\boldsymbol{\tau}^{\|}$to satisfy C2, see Propositions 5.5 and 5.6. The proposed control architecture is illustrated in Fig. 2. 


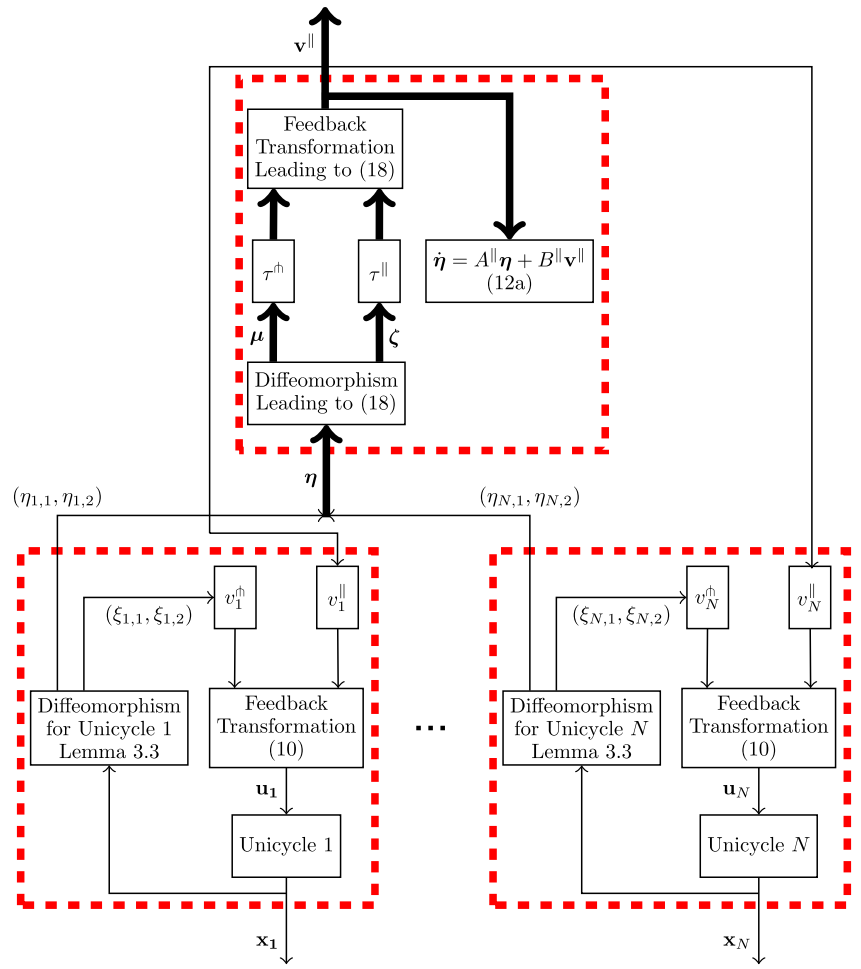

Fig. 2. Block diagram of the closed-loop system.

Remark 5.2. Instead of directly designing coordinated path following controllers, we first apply a coordinate and feedback transformation that brings the unicycles into the normal form (11) and then another that brings the multi-agent tangential dynamics (12a) into the form (18). The process of bringing the unicycles into these normal forms can involve complex, though straightforward, computations depending on the assigned paths and coordination task. However once the normal form is obtained, controller design is greatly simplified. The main challenge in implementation is the computation of (8). In general this expression does not have a closed-form solution so numerical optimization algorithms must be employed (Consolini et al., 2010). A similar computation is required to implement methods relying on Frenet-Serret frames (Ghabcheloo et al., 2007; Ghommam \& Mnif, 2009). $\triangleleft$

The next theorem explicitly addresses the component $\mathcal{P}_{+}^{\star, \mathrm{f}}:=$ $\mathcal{P}_{1,+}^{\star, \mathrm{f}} \times \cdots \times \mathcal{P}_{N,+}^{\star, \mathrm{f}} \subset \mathscr{P}^{\star}$ but a similar result can be obtained for other components of $\mathscr{P}^{\star}$.

Theorem 5.3. Fix $\overline{\mathbf{x}} \in \mathcal{P}_{+}^{\star, \mathrm{f}}$ and let $U \subset\left(\mathbb{R}^{2} \times \mathbb{S}^{1} \times \mathbb{R}\right)^{N}, \mathcal{P}_{+}^{\star, \mathrm{f}} \subset U$ be an open set containing $\overline{\mathbf{x}}$ on which the multi-unicycle system is feedback equivalent to (12). Let $\left(\overline{\boldsymbol{\eta}}, 0_{2 N}\right)=T(\overline{\mathbf{x}})$. Let (12b) be feedback equivalent, in an open set $V \subseteq T(U)$ containing $T(\overline{\mathbf{x}})$ to (18) and suppose there exist class- $\mathcal{K}_{\infty}$ functions $\rho_{1}, \rho_{2}:[0, \infty)$ $\rightarrow \mathbb{R}_{+}$such that for all $\eta \in V$

$\rho_{1}\left(\operatorname{dist}\left(\boldsymbol{\eta}, \mathcal{C}^{\star}\right)\right) \leq\|\boldsymbol{\mu}(\boldsymbol{\eta})\| \leq \rho_{2}\left(\operatorname{dist}\left(\boldsymbol{\eta}, \mathcal{C}^{\star}\right)\right)$.

If each unicycle's transversal control is given by (17), $\boldsymbol{\tau}^{\pitchfork}=F \boldsymbol{\mu}$ is such that $A+B F$ is Hurwitz, and $\boldsymbol{\tau}^{\|}$is such that for each $(\boldsymbol{\xi}(0), \eta(0)) \in V$, $(\boldsymbol{\xi}(t), \boldsymbol{\eta}(t)) \in V$ for all $t \geq 0$, then $\mathbf{x}(t) \rightarrow \mathcal{C}^{\star}$ as $t \rightarrow \infty$.

Remark 5.4. We once again stress that the existence of the function $\tilde{\beta}$ only guarantees local equivalence between the tangential dynamics (12a) and (18). Intuitively this means that the controllers of Theorem 5.3 only solve the coordinated path following problem if the unicycles are not too far from coordination at $t=0$.
Proof of Theorem 5.3. Let $\mathbf{v}^{\dagger}=F^{\pitchfork} \boldsymbol{\xi}$ be the overall transversal controller where $F^{\dagger}$ is an $N \times 2 N$ matrix composed of the gains in Eq. (17). By hypothesis, the multi-agent tangential dynamics (12a) are locally feedback equivalent to (18). In $V$ the closed-loop system is feedback equivalent to

$\dot{\zeta}=f(\zeta, \mu)+g^{\dagger}(\boldsymbol{\zeta}, \boldsymbol{\mu}) F \boldsymbol{\mu}+g^{\|}(\boldsymbol{\zeta}, \boldsymbol{\mu}) \tau^{\|}$

$\dot{\mu}=(A+B F) \mu$

$\dot{\xi}=\left(A^{\pitchfork}+B^{\pitchfork} F^{\pitchfork}\right) \xi$.

By hypothesis, solutions starting in $V$ remain in $V$ for all $t \geq$ 0 , and $A^{\dagger}+B^{\dagger} F^{\dagger}$ and $A+B F$ are Hurwitz, therefore $(\boldsymbol{\mu}, \boldsymbol{\xi})=$ $(0,0)$ is exponentially stable for the $(\boldsymbol{\mu}, \boldsymbol{\xi})$-subsystem. Under conditions (2) and (20), $(\boldsymbol{\mu}, \boldsymbol{\xi}) \rightarrow(0,0)$ is equivalent to $\mathbf{x} \rightarrow$ $e^{\star}$.

Theorem 5.3 shows that under suitable assumptions, as opposed to more general scenarios (Doosthoseini \& Nielsen, 2012; El-Hawwary \& Maggiore, 2010), for a multi-agent unicycle system the set $C^{\star}$ can be rendered locally attractive via feedback regardless of whether it is initialized on $\mathcal{P}^{\star}$.

\subsection{Velocity and position coordination}

We investigate velocity and position coordination as two special, but important, cases of coordination tasks.

Proposition 5.5 (Velocity Coordination). Given tangential dynamics (12a), a coordination function $\hat{\beta}$ satisfying the hypotheses of Proposition 4.3, and a point $\bar{\eta} \in \mathcal{C}^{\star}$, there exist a neighbourhood $V \subseteq \psi(U)$ containing $\bar{\eta}$ and a function $\tilde{\beta}: V \rightarrow \mathbb{R}^{N}$ which satisfies $\operatorname{rank} \mathrm{d} \tilde{\beta}_{\bar{\eta}}=N$. Moreover, the tangential dynamics (12a) with output $\tilde{\beta}$ yields a well-defined relative degree of $\{1, \ldots, 1\}$ at $\bar{\eta}$.

Proof. Since $\partial_{\eta_{1}} \hat{\beta} \equiv 0$ it follows rank $\mathrm{d} \hat{\beta}=\operatorname{rank} \partial_{\eta_{2}} \hat{\beta}=c \leq N$. Let $\phi: V \subseteq \psi(U) \rightarrow \mathbb{R}^{N-c}$ be a function such that $\partial_{\eta_{1}} \phi$ $\equiv 0$ and $\operatorname{rank} \partial_{\eta_{2}} \phi=N-c$. Define $\tilde{\beta}=\operatorname{col}(\phi, \hat{\beta})$. It is immediately evident that it has rank $N$ at $\bar{\eta}$. Direct calculations yield $L_{B \|} \tilde{\beta}(\boldsymbol{\eta})=\left(\partial_{\eta_{2}} \phi(\eta), \partial_{\eta_{2}} \hat{\beta}(\boldsymbol{\eta})\right)$. Since $\operatorname{rank} L_{B \|} \tilde{\beta}(\bar{\eta})=N$, the proof is complete.

Using the function $\tilde{\beta}$ of Proposition 5.5 and employing input-output feedback linearization, the tangential dynamics (12a) are locally feedback equivalent to

$\dot{\zeta}_{1}=\hat{f}\left(\zeta_{1}, \zeta_{2}, \mu\right)+\hat{g}^{\pitchfork}\left(\zeta_{1}, \zeta_{2}, \mu\right) \tau^{\pitchfork}+\hat{g}^{\|}\left(\zeta_{1}, \zeta_{2}, \mu\right) \tau^{\|}$

$\dot{\zeta}_{2}=\tau^{\|}$

$\dot{\mu}=\tau^{\pitchfork}$

where $\operatorname{col}\left(\zeta_{1}, \zeta_{2}, \boldsymbol{\mu}\right) \in \mathbb{R}^{N} \times \mathbb{R}^{N-c} \times \mathbb{R}^{c}, \operatorname{col}\left(\boldsymbol{\tau}^{\pitchfork}, \boldsymbol{\tau}^{\|}\right) \in \mathbb{R}^{c} \times \mathbb{R}^{N-c}$, $\hat{f}: V \subseteq \mathbb{R}^{2 N} \rightarrow \mathbb{R}^{N}$ is a smooth function, and $\hat{g}^{\|}: V \subseteq \mathbb{R}^{2 N} \rightarrow$ $\mathbb{R}^{N \times N-\bar{c}}$ and $\hat{g}^{\dagger}: V \subseteq \mathbb{R}^{2 N} \rightarrow \mathbb{R}^{N \times c}$ are smooth matrix-valued functions. In (22), the $\boldsymbol{\mu}$ and $\zeta_{2}$-dynamics are decoupled which allows one to design the control laws $\boldsymbol{\tau}^{\pitchfork}, \boldsymbol{\tau}^{\|}$separately, The input $\tau^{\|}$can be used to control the velocity of the coordinated unicycles along their assigned paths.

Proposition 5.6 (Position Coordination). Given the tangential $d y$ namics (12a), a coordination function $\hat{\beta}$ satisfying conditions of Proposition 4.4, and a point $\bar{\eta} \in \mathcal{C}^{\star}$, there exist a neighbourhood $V \subseteq \psi(U)$ containing $\bar{\eta}$ and a function $\tilde{\beta}: V \rightarrow \mathbb{R}^{N}$ which satisfies $\operatorname{rank} \mathrm{d} \tilde{\beta}_{\bar{\eta}}=N$. Moreover, the tangential dynamics (12a) with output $\tilde{\beta}$ yield a well-defined vector relative degree of $\{2, \ldots, 2\}$ at $\bar{\eta}$.

Proof. Let $\phi: V \subseteq \psi(U) \rightarrow \mathbb{R}^{N-c}$ be a function such that $\partial_{\eta_{2}} \phi \equiv$ 0 and rank $\partial_{\eta_{1}} \phi=N-c$. Define $\tilde{\beta}=\operatorname{col}(\phi, \beta)$. Since $\partial_{\eta_{2}} \hat{\beta} \equiv 0$ it follows rank $\mathrm{d} \hat{\beta}=\operatorname{rank} \partial_{\eta_{1}} \hat{\beta}=c \leq N$. As a result $\tilde{\beta}$ has rank 
$N$ at $\bar{\eta}$. Simple calculations give $L_{B \|} \tilde{\beta}=\mathbf{0}_{N}$ and $L_{B \|} L_{A} \|_{\eta} \tilde{\beta}(\boldsymbol{\eta})=$ $\left(\partial_{\eta_{1}} \phi(\boldsymbol{\eta}), \partial_{\eta_{1}} \hat{\beta}(\boldsymbol{\eta})\right)$ which has rank $N$ at $\overline{\boldsymbol{\eta}}$.

The function $\tilde{\beta}$ of Proposition 5.6 can be used to feedback linearize the tangential dynamics (12a) to obtain

$$
\begin{aligned}
& \dot{\zeta}_{1}=\zeta_{2} \\
& \dot{\zeta}_{2}=\boldsymbol{\tau}^{\|} \\
& \dot{\mu}_{1}=\boldsymbol{\mu}_{2} \\
& \dot{\boldsymbol{\mu}}_{2}=\boldsymbol{\tau}^{\pitchfork}
\end{aligned}
$$

where $\operatorname{col}\left(\boldsymbol{\zeta}_{1}, \boldsymbol{\zeta}_{2}, \boldsymbol{\mu}_{1}, \boldsymbol{\mu}_{2}\right) \in \mathbb{R}^{N-c} \times \mathbb{R}^{N-c} \times \mathbb{R}^{c} \times \mathbb{R}^{c}, \operatorname{col}\left(\boldsymbol{\tau}^{\pitchfork}, \boldsymbol{\tau}^{\|}\right) \in$ $\mathbb{R}^{c} \times \mathbb{R}^{N-c}$. In (23), the $\left(\boldsymbol{\mu}_{1}, \boldsymbol{\mu}_{2}\right)$ and $\left(\zeta_{1}, \zeta_{2}\right)$-dynamics are decoupled. As a result, the control inputs $\boldsymbol{\tau}^{\pitchfork}$ and $\boldsymbol{\tau}^{\|}$can be designed separately. The input $\boldsymbol{\tau}^{\|}$can be used to control the position and velocity of the coordinated unicycles along their assigned paths.

\subsection{Semi-distributed stabilization of a linear-affine coordination set}

Here we consider a specific, yet useful, choice of linear-affine coordination function $\hat{\beta}(\boldsymbol{\eta})$. Consider a coordination constraint in which every two consecutive unicycles must maintain a constant arc-length separation, i.e., $\eta_{i+1,1}-\eta_{i, 1}-b_{i, 1}=0, i \in \mathbf{N}-$ 1 where $b_{i, 1} \in \mathbb{R}$. Moreover, it is required that once all the agents are in formation they all move with a desired velocity $v_{d}>0$, i.e., $\eta_{i, 2}-v_{d}=0, i \in \mathbf{N}$. This particular coordinated path following problem is the same as the formation control problem investigated in Ren (2007). We can represent this special coordination function as a linear-affine function. In order to do so we add a redundant constraint $\eta_{N, 1}-\eta_{1,1}-b_{N, 1}=0$ and define the coordination function $\hat{\beta}(\boldsymbol{\eta})=A \boldsymbol{\eta}+b$ as $\hat{\beta}(\boldsymbol{\eta})=A \boldsymbol{\eta}+b$ as $b_{1}=\left(b_{1,1}, \ldots, b_{N, 1}\right), b_{2}=-v_{d} 1_{N}$,

$A_{1}=\left[\begin{array}{c}A_{11} \\ \mathbf{0}_{N}\end{array}\right], \quad A_{2}=\left[\begin{array}{l}\mathbf{0}_{N} \\ \mathbf{I}_{N}\end{array}\right]$,
$A_{11}=\left[\begin{array}{ccccc}1 & -1 & 0 & \cdots & 0 \\ 0 & 1 & -1 & \cdots & 0 \\ \vdots & \vdots & \vdots & \vdots & \vdots \\ -1 & 0 & 0 & \cdots & 1\end{array}\right]$.

Assumption 3. The numbering assigned to the agents, the prescribed velocity $v_{d}$, the vector $b_{1}$, and a number $k>0$ are known to each unicycle.

The following less restrictive assumption replaces Assumption 2.

Assumption 4. The communication graph $\mathscr{G}$ of the multi-agent unicycle system is rooted out-branching.

Lemma 5.7. Let $\mathscr{G}$ be a weighted and directed graph on $N$ vertices and let $L$ be its Laplacian matrix. If $H:=-L \mathbf{U}_{N}$ then $H A_{11}=-L$ where $A_{11}$ is given by (24).

Proof. Since $H=-L \mathbf{U}_{N}$ we must show that $-L \mathbf{U}_{N} A_{11}=-L$. Let $L_{i}$ denote the $i$ th column of $-L$, for $i \in \mathbf{N}$. Then $-L \mathbf{U}_{N} A_{11}=$ $\left[\begin{array}{llll}\sum_{i=2}^{N}-L_{i} & L_{2} & \cdots & L_{N}\end{array}\right]$. A well-known property of the Laplacian matrix is that its columns sum to zero, i.e, $L_{1}+L_{1}+\cdots+L_{N}=$ 0 (Ren, Beard, \& Atkins, 2007). So, we can write the first column, $L_{1}$, in terms of other columns $L_{1}=-\left(L_{2}+L_{3}+\cdots+L_{N}\right)$. As a result

$-L U A_{11}=\left[\begin{array}{llll}L_{1} & L_{2} & \cdots & L_{N}\end{array}\right]=-L$.
Lemma 5.8. If each path $\gamma_{i}, i \in \mathbf{N}$ is non-closed and the communication graph $\mathscr{G}$ of the multi-unicycle system is rooted out-branching then the control law

$$
\begin{aligned}
v_{i}^{\|}= & \sum_{j=1}^{N} w_{i j}\left(\eta_{j, 1}-\eta_{i, 1}-\frac{(j-i)}{|j-i|} \sum_{p=\min \{i, j\}}^{\max \{i, j\}-1} b_{1, p}\right) \\
& -k\left(\eta_{i, 2}-v_{d}\right)
\end{aligned}
$$

where $w_{i j}$ are entries of the adjacency matrix $W(\mathscr{G})$ and $k>0$, renders $\mathcal{C}^{\star}$ globally asymptotically stable relative to $\mathcal{P}^{\star}$.

Remark 5.9. Control law (25) is adapted from Ren (2007). Similar control laws can be found in swarming problems (Jin \& Gao, 2008). We present (25) to demonstrate the use of existing distributed controllers in specific cases of coordination.

Proof of Lemma 5.8. We view the coordination function as an error function $\mathbf{e}=A \eta+b$. Partitioning $\mathbf{e}=\left(\mathbf{e}_{1}, \mathbf{e}_{2}\right)$ in accordance with the linear coordination function we have

$\mathbf{e}_{1}=A_{11} \boldsymbol{\eta}_{1}+b_{1}$

$\mathbf{e}_{2}=\eta_{2}+b_{2}$.

The closed-loop error dynamics resulting from applying the control law (25) to the dynamics on $\psi(U)$ given in (12a) are

$\left[\begin{array}{c}\dot{\mathbf{e}}_{1} \\ \dot{\mathbf{e}}_{2}\end{array}\right]=\left[\begin{array}{cc}\mathbf{0}_{N} & A_{11} \\ H & -k \mathbf{I}_{N}\end{array}\right]\left[\begin{array}{l}\mathbf{e}_{1} \\ \mathbf{e}_{2}\end{array}\right]=: E \mathbf{e}$.

An immediate result is that 0 is an eigenvalue of matrix $E$ since $A_{11}$ has rank $N-1$. However, we do not yet know the algebraic multiplicity of the eigenvalue 0 . In the following we find the remaining eigenvalues of the matrix $E$. Let $\lambda$ be an eigenvalue of $E$ with associated eigenvector $(x, y) \in \mathbb{C}^{N} \times \mathbb{C}^{N}$. The relation between eigenvalues and eigenvectors of the matrix $E$ is

$A_{11} y=\lambda x$

$H x-k y=\lambda y$.

Suppose that $\lambda \neq 0$. Combining the equations in (28) and using the result from Lemma 5.7 we obtain

$\frac{1}{\lambda} \underbrace{H A_{11}}_{-L} y-k y=\lambda y \Rightarrow-L y=(k+\lambda) \lambda y$.

The Laplacian $L$ has eigenvalues $\left\{v_{1}, \ldots, v_{N}\right\}$, one of which is zero. From (29) we deduce that $(k+\lambda) \lambda$ is an eigenvalue of $-L$. This yields the following $N$ equations

$\left(k+\lambda_{i}\right) \lambda_{i}=v_{i}, \quad i \in\{1, \ldots, N\}$.

There are $2 N$ solutions to the above equations

$\lambda_{i}^{ \pm}=\frac{-k \pm \sqrt{k^{2}+4 v_{i}}}{2}, \quad i \in\{1, \ldots, N\}$.

Since we assumed that $\lambda \neq 0$ in (29), the solution $\lambda_{1}^{+}=0$ corresponding to $v_{i}=0$ is not an allowable solution. However, since we already know that 0 is an eigenvalue of $E$; it is just not obtained from solving above equation. Since $\mathscr{G}$ is rooted outbranching, the algebraic and geometric multiplicity of $v_{i}=0$ is 1 . Therefore, all the $\lambda_{i}^{ \pm}$'s obtained from above equation have negative real parts, since $\lambda_{1}^{-}=-k$ and rest of $v_{i}$ have negative real parts. As a result, the eigenvalue 0 has geometric and algebraic multiplicity of 1 . Using standard spectral theory, there exists a $2 \mathrm{~N} \times 2 \mathrm{~N}$ matrix $V$ such that the similarity transform $E \mapsto V^{-1} E V$ yields the Jordan form $E_{J F}=\operatorname{diag}\left(0, J_{1}, \ldots, J_{r}\right)$ where $r$ is the number of distinct eigenvalues of $E$ and each Jordan block $J_{i}$ has the form $\lambda_{i} I+N_{i}$, 
where $N_{i}$ is a nilpotent matrix in Jordan form and $\operatorname{Re}\left(\lambda_{i}\right)<0$. We therefore have that

$$
\begin{aligned}
\lim _{t \rightarrow \infty} \mathrm{e}^{E t} & =\lim _{t \rightarrow \infty} V \mathrm{e}^{E_{J F} t} V^{-1}=V \operatorname{diag}(1,0, \ldots, 0) V^{-1} \\
& =p_{1} q_{1}
\end{aligned}
$$

where $p_{1}$ is the first column of $V$ and $q_{1}$ is the first row of $V^{-1}$. It is easy to see that $p_{1}$ and $q_{1}$ are, respectively, the right and left eigenvectors of $E$ associated with the eigenvalue 0 . Thus, for any $e(0) \in \mathbb{R}^{2 N}$, the solution to (27) can be written.

$$
\lim _{t \rightarrow \infty} \mathbf{e}(t)=\left(q_{1} \mathbf{e}(0)\right) p_{1} .
$$

Direct calculations reveal that the following are, respectively, the right and left eigenvectors of the matrix $E$ associated with the zero eigenvalue

$p_{1}=\left(0_{N-1}, 1,0_{N}\right) \quad q_{1}^{\top}=\left(1_{N}, 0_{N}\right)$.

Therefore (30) is given by

$\lim _{t \rightarrow \infty} \mathbf{e}(t)=\operatorname{col}\left(0_{N-1}, e_{1,1}(0)+\cdots+e_{N, 1}(0), 0_{N}\right)$.

Since the errors are not independent, they satisfy, for all $t \geq 0$, $e_{1,1}(t)+\cdots+e_{N, 1}(t)=0 \quad \forall t \geq 0$. Thus $\lim _{t \rightarrow \infty} \mathbf{e}(t)=0_{2 N}$.

We now consider the case when all paths are closed, i.e., $\eta_{i, 1} \in$ $\mathbb{R} \bmod L_{i}$. In order to define a similar formation coordination constraint in this case we define $\eta_{i, 1}^{\prime}:=\frac{2 \pi}{L_{i}} \eta_{i, 1}$. The variable $\eta_{i, 1}^{\prime}$ belongs to $[0,2 \pi)$ so we can view it as an angular variable. Accordingly, we define $\eta_{i, 2}^{\prime}:=\frac{2 \pi}{L_{i}} \eta_{i, 2}$. Thus, the coordination constraint (24) imposes that two consecutive unicycles maintain a constant angular separation, i.e., $\eta_{i+1,1}^{\prime}-\eta_{i, 1}^{\prime}-b_{i, 1}=0, i \in \mathbf{N}-\mathbf{1}$ where $b_{i, 1} \in[0,2 \pi)$. Moreover, the formation must move with a common velocity $\omega_{d}>0$, i.e., $\eta_{i, 2}^{\prime}-\omega_{d}=0, i \in \mathbf{N}$.

Corollary 5.10. Suppose $\gamma_{i}, i \in \mathbf{N}$ are closed paths and the communication graph $\mathscr{G}$ of the multi-unicycle system is rooted out-branching then the control law

$$
\begin{aligned}
v_{i}^{\|}= & \sum_{j=1}^{N} w_{i j} \sin \left(\eta_{j, 1}^{\prime}-\eta_{i, 1}^{\prime}-\frac{(j-i)}{|j-i|} \sum_{p=\min \{i, j\}}^{\max \{i, j\}-1} b_{i, p}\right) \\
& -k\left(\eta_{i, 2}^{\prime}-v_{d}\right)
\end{aligned}
$$

where $w_{i j}$ are the entries of $W(\mathscr{G})$ and $k>0$, renders $\mathcal{C}^{\star}$ locally exponentially stable relative to $\mathcal{P}^{\star}$.

Proof. The closed-loop error dynamics resulting from applying the control law (32) to the dynamics on $\mathcal{P}^{\star}$ given in (12a) are

$\dot{\mathbf{e}}_{1}=\mathbf{e}_{2}$

$\dot{\mathbf{e}}_{2}=H \sin \left(\mathbf{e}_{1}\right)-k \mathbf{I}_{N} \mathbf{e}_{2}$

where $\sin \left(\mathbf{e}_{1}\right):=\sin \left(e_{1,1}\right), \ldots, \sin \left(e_{1, N}\right)$. Linearizing the above closed-loop about $\left(\mathbf{e}_{1}, \mathbf{e}_{2}\right)=(0,0)$ results in error dynamics given in (27). Lemma 5.8 shows that $(0,0)$ is globally exponentially stable for error dynamics $(27)$. Therefore $(0,0)$ is locally exponentially stable for (33).

Remark 5.11. When all the paths are closed the multi-agent path following manifold is diffeomorphic to $\mathbb{T}^{N} \times \mathbb{R}^{N}$. Since $\mathbf{v}^{\|}$is $2 \pi$ periodic in its $N$ arguments it is a continuous function that is defined globally on the multi-agent path following manifold.

\section{Experimental implementation}

We experimentally verify our results using two TurtleBots built by Clearpath Inc. The robots have a maximum translational speed of $65 \mathrm{~cm} / \mathrm{s}$ and maximum rotational speed of $\pi \mathrm{rad} / \mathrm{s}$. Each robot is controlled using the Robot Operating System (R.O.S.) running on an Intel Atom Notebook with Linux. An Indoor Positioning System (I.P.S.) using NaturalPoint OptiTrack provides the states $\left(x_{i}, y_{i}, \theta_{i}\right)$ of robot $i$ over WiFi at $100 \mathrm{~Hz}$. The state $v_{i}$ of robot $i$ is obtained by integrating the control input $u_{i, 1}$.

\subsection{Coordination specification}

The robots are assigned circular paths

$\gamma_{i}=\left\{\mathbf{y}_{i} \in \mathbb{R}^{2}:\left\|\mathbf{y}_{i}\right\|_{2}^{2}-r_{i}^{2}=0\right\}, \quad i \in \mathbf{2}$,

$r_{1}=1.1, r_{2}=0.75 \mathrm{~m}$. These paths satisfy inequality (2) of Assumption 1 with $\rho_{i, 1}, \rho_{i, 2}$, taken as identity functions. Since both robots can communicate with each other the communication graph is fully connected and $\mathbf{C 3}$ is trivially satisfied. The coordination specification $\mathbf{C 1}$ is that the two robots be on opposite sides of their respective circles. Such a coordination specification can arise in patrolling applications because it results in better coverage of an area. For $\mathbf{C} 2$ we require that robots 1 and 2 move with a prescribed angular velocity $\omega_{d}=.03 \mathrm{rad} / \mathrm{s}$. Note that $\eta_{i, 1}^{\prime}=\frac{\eta_{i, 1}}{r_{i}}, \eta_{i, 2}^{\prime}=\frac{\eta_{i, 2}}{r_{i}}, i \in \mathbf{2}$. Thus the linear-affine coordination function (24) becomes

$\beta\left(\boldsymbol{\eta}^{\prime}\right)=\left[\begin{array}{cccc}1 & -1 & 0 & 0 \\ -1 & 1 & 0 & 0 \\ 0 & 0 & 1 & 0 \\ 0 & 0 & 0 & 1\end{array}\right]\left[\begin{array}{l}\eta_{1,1}^{\prime} \\ \eta_{2,1}^{\prime} \\ \eta_{1,2}^{\prime} \\ \eta_{2,2}^{\prime}\end{array}\right]-\left[\begin{array}{c}\pi \\ -\pi \\ \omega_{d} \\ \omega_{d}\end{array}\right]$.

Using Proposition 4.2, condition (1), we immediately find that $\mathcal{C}=$ $\mathcal{C}^{\star}$ and $\operatorname{dim} \mathcal{C}^{\star}=2$. After bringing each robot into the normal form (11), the transversal control laws are taken to be (17) with the high gains $k_{i, 1}=30, k_{i, 2}=20, i \in \mathbf{2}$ to make the robots approach their paths quickly. The coordination control laws are obtained using Corollary 5.10

$v_{1}^{\|}=w_{12} \sin \left(\eta_{2,1}^{\prime}-\eta_{1,1}^{\prime}-\pi\right)-k\left(\eta_{1,2}^{\prime}-\omega_{d}\right)$

$v_{2}^{\|}=w_{21} \sin \left(\eta_{1,1}^{\prime}-\eta_{2,1}^{\prime}-\pi\right)-k\left(\eta_{2,2}^{\prime}-\omega_{d}\right)$

where $w_{12}, w_{21}, k>0$. The communication graph weights are treated as controller gains and taken to be $w_{21}=w_{12}=k=$ 10. These gains are smaller, relatively, than the path following controller gains because we prioritize convergence to the paths over coordination.

Experimental output trajectories are shown in Fig. 3(a). The path error for each unicycle is computed as

$e_{i, \mathrm{PF}}:=\sqrt{x_{i}^{2}+y_{i}^{2}}-r_{i}, \quad i \in\{1,2\}$

and shown in Fig. 3(b). Fig. 4(a) displays the coordination error, $e_{\mathrm{C} 1}:=\eta_{1,1}^{\prime}-\eta_{2,1}^{\prime}-\pi$, expressed in radians, converging to zero. Fig. 4(b) shows that each robot's angular velocity error, $e_{i, \mathrm{C} 2}:=$ $\eta_{i, 2}^{\prime}-\omega_{d}, i \in \mathbf{2}$, converges to zero quickly. Fig. 5(a) and (b) shows the control effort required in these experiments.

\subsection{Switching coordination specifications}

A distinguishing feature of the proposed controllers is that the paths $\gamma_{1}$ and $\gamma_{2}$ are invariant for robots 1 and 2 . Therefore, if the coordination specification changes we expect the robots to remain on their assigned paths, before eventually re-coordinating. In this experiment we ask that robot 1 initially be phase shifted by $\frac{\pi}{2}$ radians from robot 2 and after $240 \mathrm{~s}$ the phase difference changes to $\pi$.

Fig. 6(a) shows experimental output trajectories of the robots. When the coordination specification is changed the robots are 


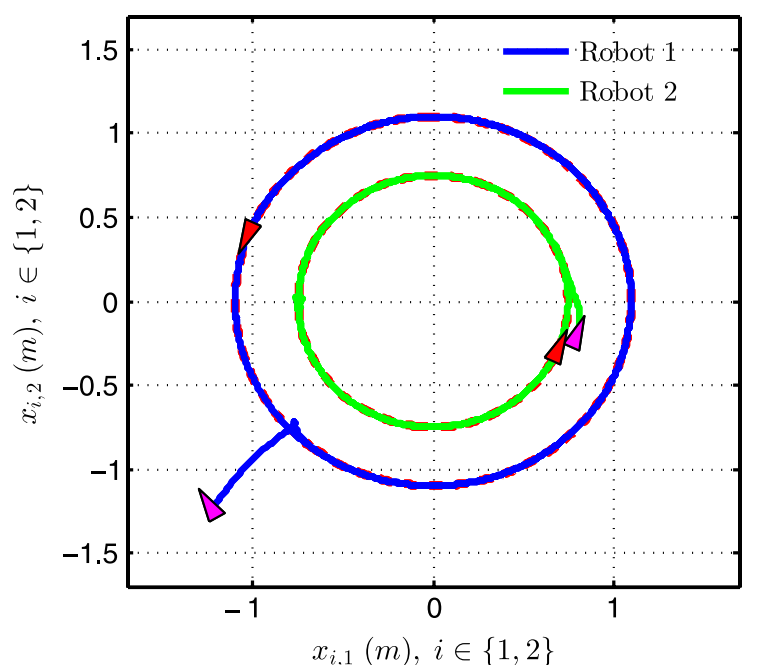

(a) Output trajectories: initial positions and final positions of robots are indicated, respectively, by magenta and red triangles.

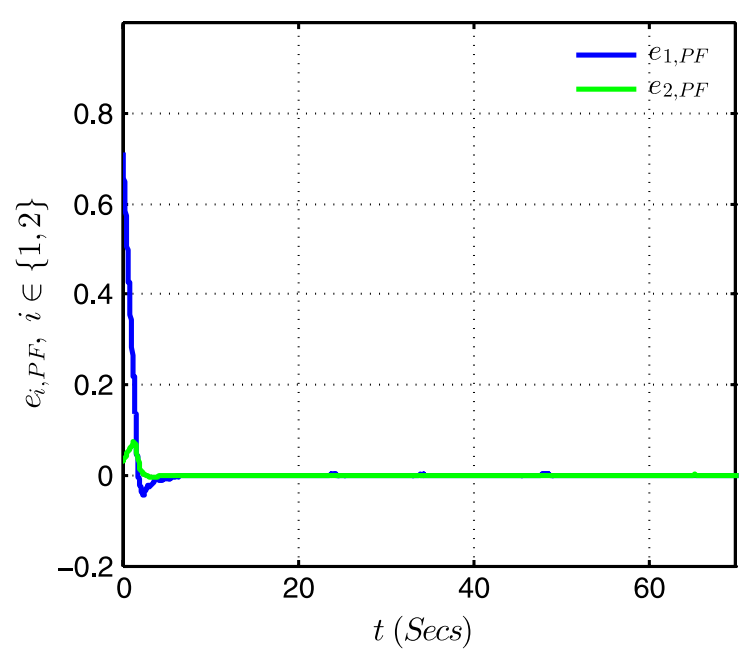

(b) Path following error for robots 1 and 2 .

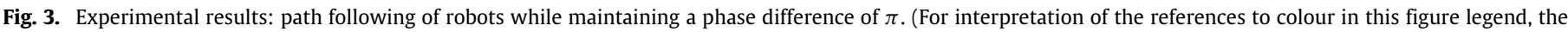
reader is referred to the web version of this article.)

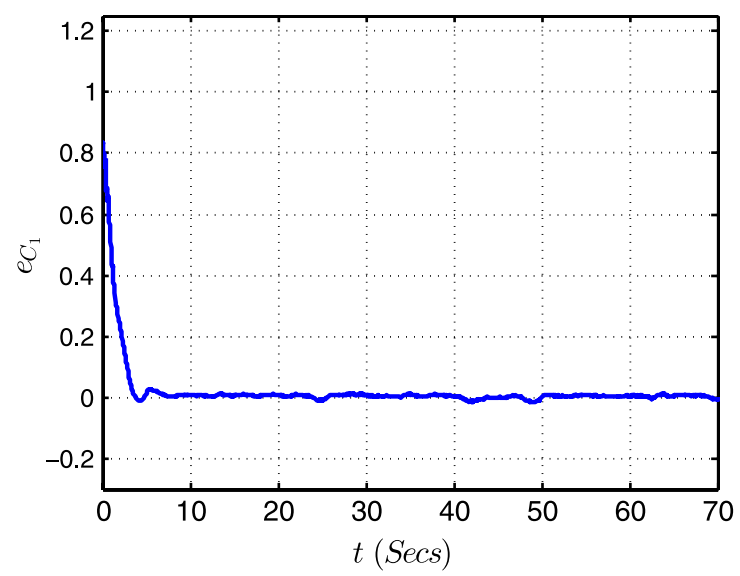

(a) Coordination error

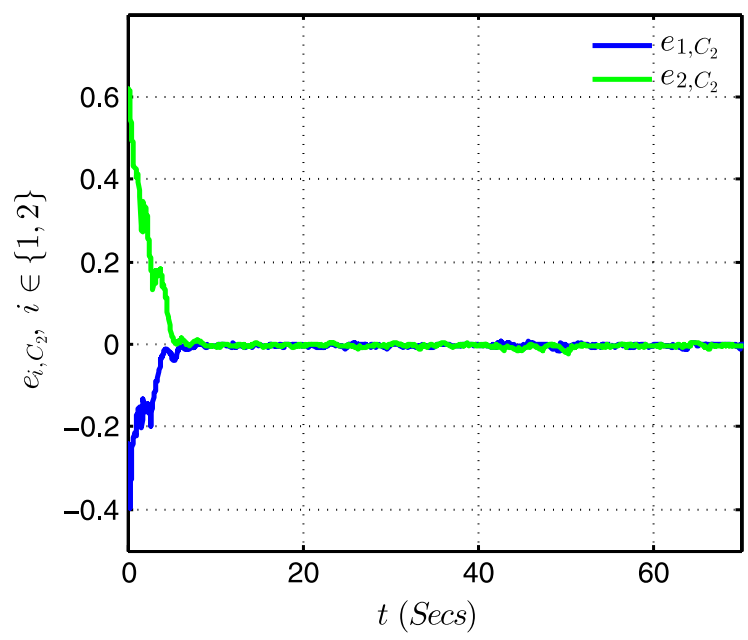

(b) Angular velocity error.

Fig. 4. Experimental results: coordination and angular velocity error while maintaining a phase difference of $\pi$.

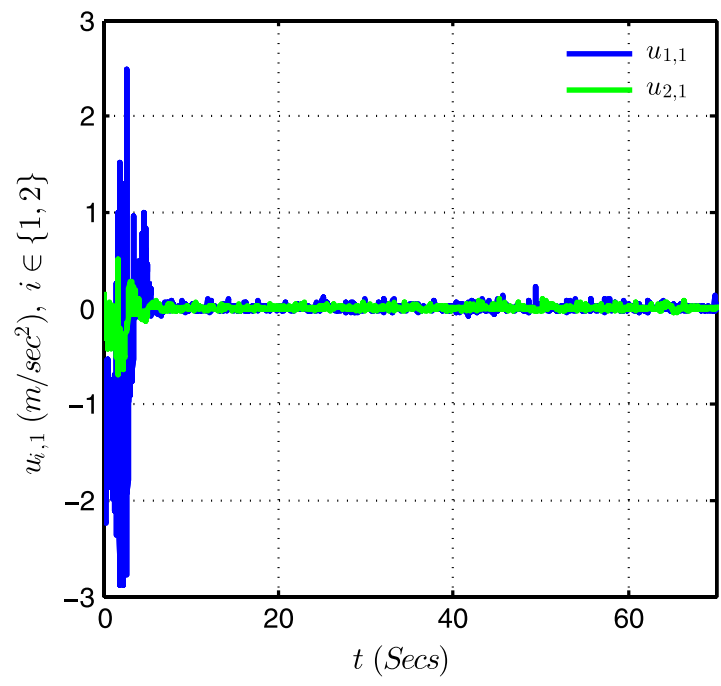

(a) Control signals $u_{i, 1}, i \in \mathbf{2}$.

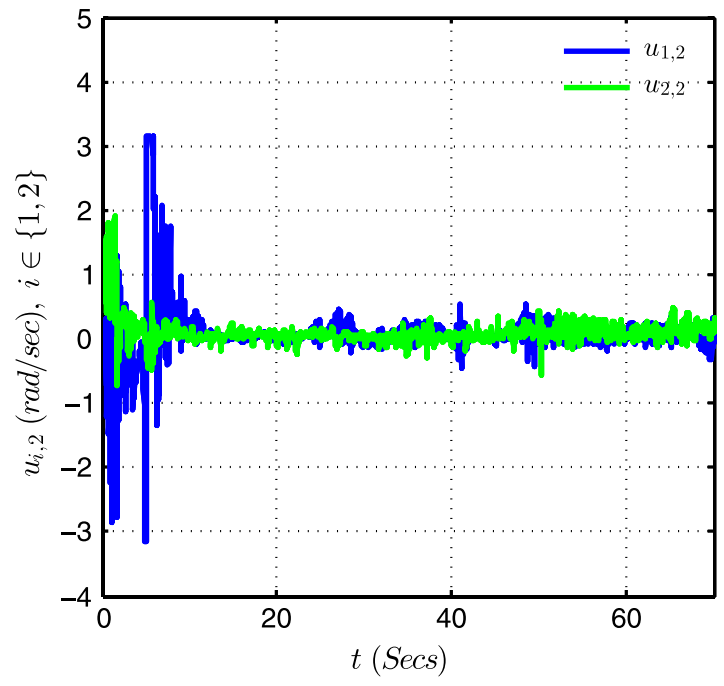

(b) Control signals $u_{i, 2}, i \in \mathbf{2}$.

Fig. 5. Experimental results: control signals $u_{i, j}, i, j \in \mathbf{2}$, while maintaining a phase difference of $\pi$. 


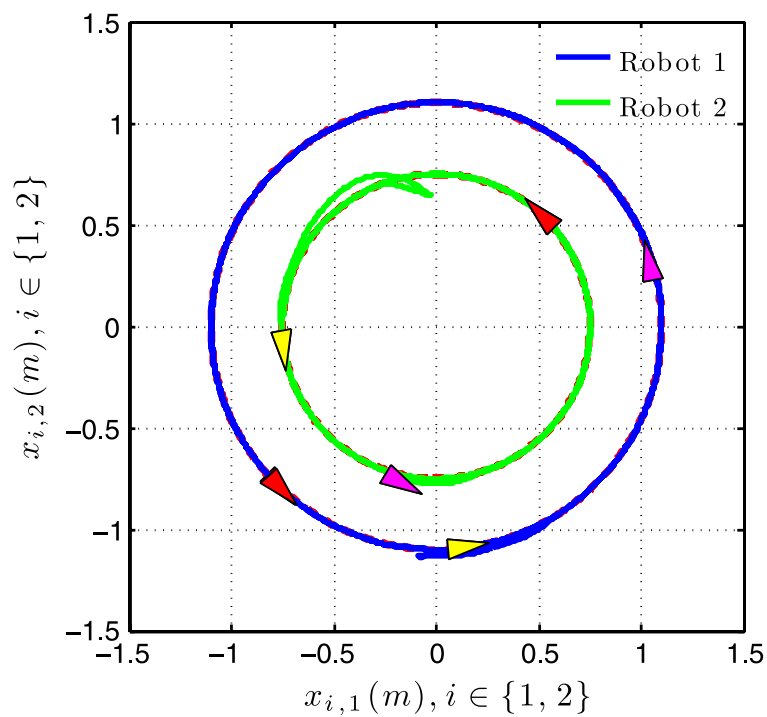

(a) Output trajectories for experiment 2: initial positions, positions before change of coordination task, and final positions of robots are indicated, respectively, by magenta, yellow, and red triangles.

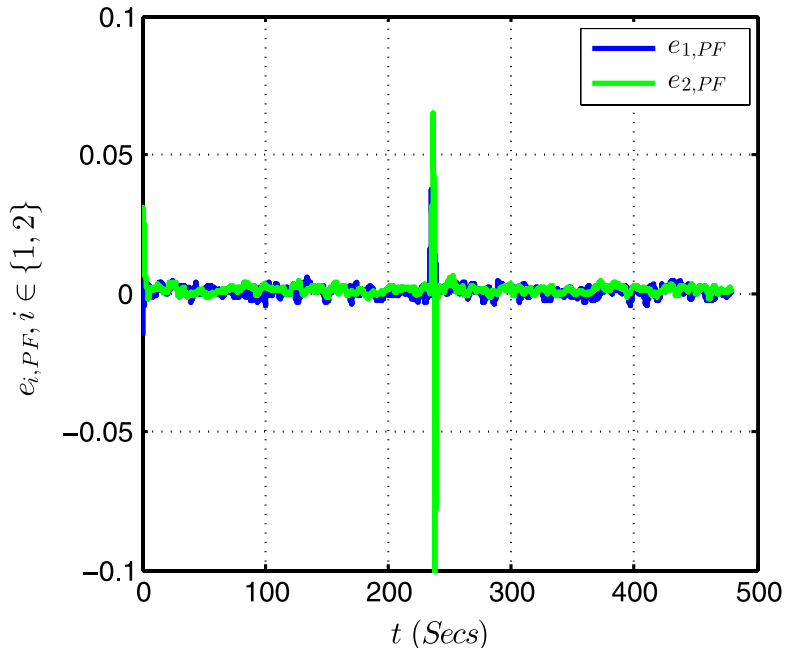

(b) Path following error for robots 1 and 2 .

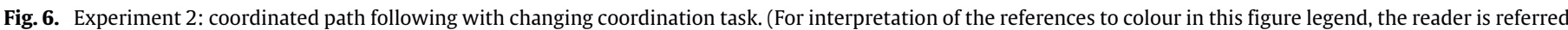
to the web version of this article.)

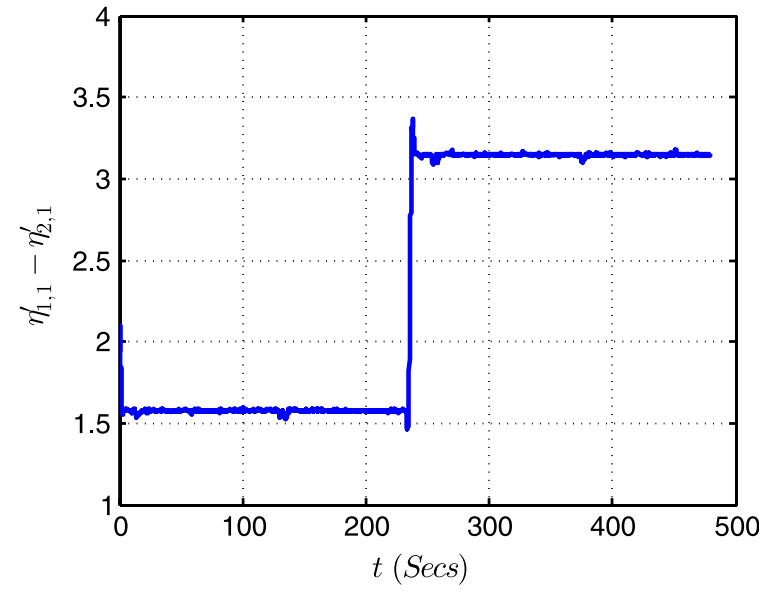

(a) Phase difference of robots on circular paths.

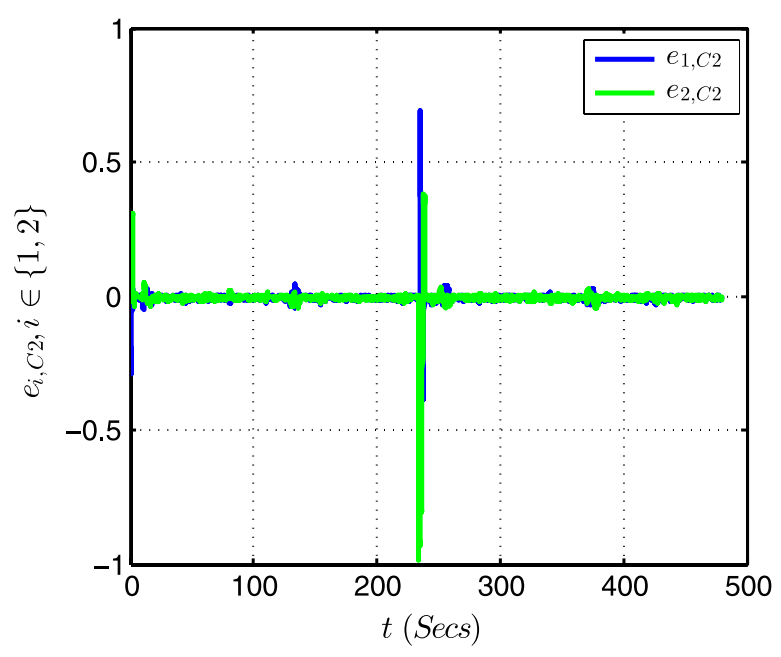

(b) Angular velocity error.

Fig. 7. Experiment 2: phase difference and angular velocity error when the coordination specification changes.

expected to stay on their paths. In this experiment the robots actually leave their paths because their forward velocities, $v_{i}$, pass through 0 which are singularities. The control signals remain bounded because we enforce actuator constraints $\left|u_{i, 2}\right| \leq \frac{\pi}{2}$ and $\left|v_{i}\right| \leq 0.5$, once the robots' forward velocities become nonzero, the nominal controllers take over and drive them back to their paths as shown in Fig. 6(b). Fig. 7(a) plots the difference in phase $\eta_{1,1}^{\prime}-\eta_{2,1}^{\prime}$. It is initially $\pi / 2$ and at $t=240$ it increases to $\pi$. Fig. 7 (b) shows that each robot's angular velocity error, $e_{i, C 2}$, converges to zero quickly. Figs. 8(a) and (b) illustrate control signals $u_{i, 1}$ and $u_{i, 2}$ for $i \in\{1,2\}$, respectively.

\section{Conclusions and future research}

We have treated the coordinated path following problem for a multi-agent system of dynamic unicycles as an instance of nested set stabilization yielding a local solution with path and coordination invariance. Each unicycle is transformed to a normal form, valid in a neighbourhood of each component of the path following manifold, which decouples the design of path following and coordination controllers. A broad class of coordination specifications are considered. Centralized control laws that locally solve the coordination portion of the problem are proposed and experimentally verified.

Future research entails taking into account communication constraints for general coordination constraints, developing singularity avoidance methods and employing collision avoidance techniques in conjunction with the results of this paper.

\section{Acknowledgements}

The authors thank Prof. Steven L. Waslander and Prof. Baris Fidan for providing access to lab facilities and Adeel Akhtar for helping with the experimental setup. 


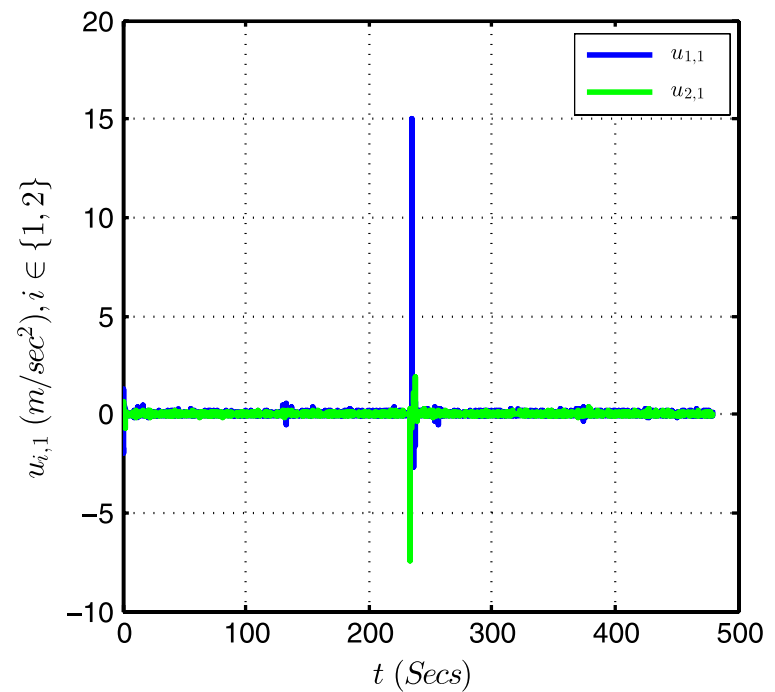

(a) Control signals $u_{i, 1}, i \in \mathbf{2}$.

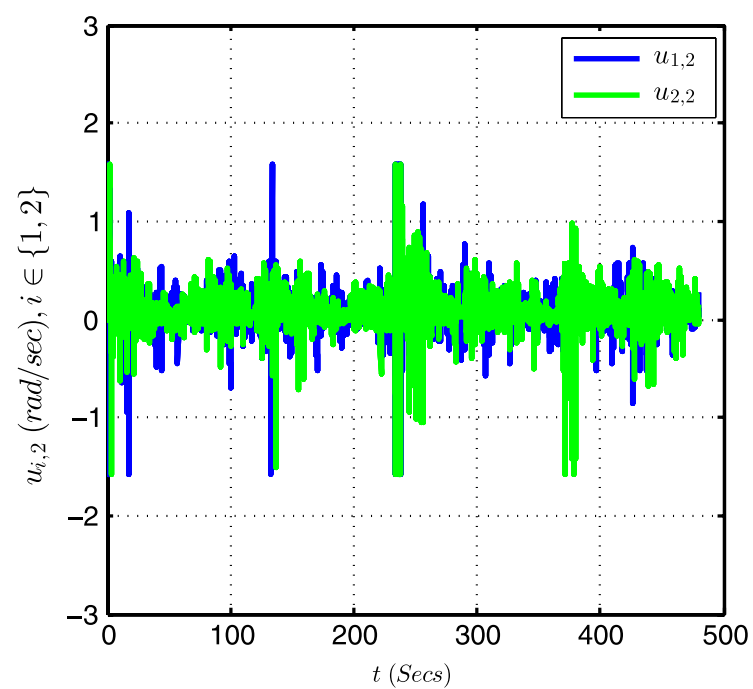

(b) Control signals $u_{i, 2}, i \in \mathbf{2}$.

Fig. 8. Experiment 2: control signals $u_{i, j}, i, j \in \mathbf{2}$ when the coordination specification changes.

\section{References}

Aguiar, A. P., \& Hespanha, J. P. (2007). Trajectory-tracking and path-following of underactuated autonomous vehicles with parametric uncertainty. IEEE Transactions on Automatic Control, 52(8), 1362-1378.

Akhtar, A., Nielsen, C., \& Waslander, S. L. (2015). Path following using dynamic transverse feedback linearization for car-like robots. IEEE Transactions on Robotics, 31(2), 269-279.

Bhat, S. P., \& Bernstein, D. S. (2000). A topological obstruction to continuous global stabilization of rotational motion and the unwinding phenomenon. Systems $\mathcal{E}^{\prime}$ Control Letters, 39(1), 63-70.

Bullo, F., \& Lewis, A. D. (2005). Geometric control of mechanical systems. Springer.

Chen, Y., \& Tian, Y. (2011). A curve extension design for coordinated path following control of unicycles along given convex loops. International Journal of Control, 84(10), 1729-1745.

Chen, J., Wang, L., \& Xiao, F. (2009). Velocity-consensus control for networks of multiple double-integrators. In Joint 48th IEEE conference on decision and control and 28th chinese control conference (pp. 2052-2057). IEEE.

Consolini, L., Maggiore, M., Nielsen, C., \& Tosques, M. (2010). Path following for the PVTOL aircraft. Automatica, 46(8), 1284-1296.

Dačić, D. B., Nešić, D., \& Kokotović, P. (2007). Path-following for nonlinear systems with unstable zero dynamics. IEEE Transactions on Automatic Control, 52(3), 481-487.

Doosthoseini, A., \& Nielsen, C. (2012). Stability problems associated with the transverse feedback linearization normal form. In Proceedings of 25th IEEE canadian conference on electrical \&' computer engineering (pp. 1-4).

Doosthoseini, A., \& Nielsen, C. (2013). Coordinated path following for a multi-agent system of unicycles. In Proceedings of 52nd IEEE conference on decision and control (pp. 2894-2899) 10-13 Dec.

Do, K. D., \& Pan, J. (2007). Nonlinear formation control of unicycle-type mobile robots. Robotics and Autonomous Systems, 55(3), 191-204.

Dorogovtsev, S., Goltsev, A., \& Mendes, J. (2008). Critical phenomena in complex networks. Reviews of Modern Physics, 80, 1275-1335.

Dörfler, F., \& Bullo, F. (2014). Synchronization in complex networks of phase oscillators: A survey. Automatica, 50(6), 1539-1564.

El-Hawwary, M. I., \& Maggiore, M. (2008). Global path following for the unicycle and other results. In American control conference (pp. 3500-3505). IEEE.

El-Hawwary, M. I., \& Maggiore, M.(2010). Reduction principles and the stabilization of closed sets for passive systems. IEEE Transactions on Automatic Control, 55(4), 982-987.

El-Hawwary, M. I., \& Maggiore, M. (2013). Distributed circular formation stabilization for dynamic unicycles. IEEE Transactions on Automatic Control, 58(1), 149-162.

Encarnaçao, P., \& Pascoal, A. (2001). Combined trajectory tracking and path following: an application to the coordinated control of autonomous marine craft. In Proceedings of the 40th IEEE conference on decision and control, volume 1 (pp. 964-969).

Ghabcheloo, R., Pascoal, A., Silvestre, C., \& Kaminer, I. (2006). Coordinated path following control of multiple wheeled robots using linearization techniques. International Journal of Systems Science, 37(6), 399-414.

Ghabcheloo, R., Pascoal, A., Silvestre, C., \& Kaminer, I. (2007). Nonlinear coordinated path following control of multiple wheeled robots with bidirectional communication constraints. International Journal of Adaptive Control and Signal Processing, 21(2-3), 133-157.
Ghommam, J., \& Mnif, F. (2009). Coordinated path-following control for a group of underactuated surface vessels. IEEE Transactions on Industrial Electronics, 56(10), 3951-3963.

Guillemin, V., \& Pollack, A. (2010). Differential topology. American Mathematical Society (RI).

Ihle, I. F., Arcak, M., \& Fossen, T. I. (2007). Passivity-based designs for synchronized path-following. Automatica, 43(9), 1508-1518.

Jin, D., \& Gao, L. (2008). Stability analysis of a double integrator swarm model related to position and velocity. Transactions of the Institute of Measurement and Control, 30(3-4), 275-293.

Lapierre, L., Soetanto, D., \& Pascoal, A. (2004). Coordinated motion control of marine robots. In Proceedings of the 6th IFAC conference on manoeuvering and control of marine craft.

Lee, J. M. (2002). Introduction to smooth manifolds. New York: Springer.

Nielsen, C., Fulford, C., \& Maggiore, M. (2010). Path following using transverse feedback linearization: Application to a maglev positioning system. Automatica, 46(3), 585-590

Nielsen, C., \& Maggiore, M. (2004). Maneuver regulation via transverse feedback linearization: Theory and examples. In Proceedings of the IFAC symposium on nonlinear control systems (NOLCOS), Stuttgart, Germany (pp. 59-66).

Nielsen, C., \& Maggiore, M. (2008). On local transverse feedback linearization. SIAM Journal on Control and Optimization, 47(5), 2227-2250.

Paley, D. A., Leonard, N. E., \& Sepulchre, R. (2008). Stabilization of symmetric formations to motion around convex loops. Systems \& Control Letters, 57(3), 209-215.

Ren, W. (2007). Consensus strategies for cooperative control of vehicle formations. Control Theory \&'Applications, IET, 1(2), 505-512.

Ren, W., Beard, R. W., \& Atkins, E. M. (2007). Information consensus in multivehicle cooperative control. Control Systems, IEEE, 27(2), 71-82.

Scardovi, L., Sarlette, A., \& Sepulchre, R. (2007). Synchronization and balancing on the n-torus. Systems \& Control Letters, 56(5), 335-341.

Sepulchre, R., Paley, D., \& Leonard, N. E. (2007). Stabilization of planar collective motion: All-to-all communication. IEEE Transactions on Automatic Control, 52(5).

Skjetne, R., Fossen, T. I., \& Kokotović, P. V. (2004). Robust output maneuvering for a class of nonlinear systems. Automatica, 40(3), 373-383.

Tomlin, C. J., \& Sastry, S. S. (1998). Switching through singularities. Systems \& Control Letters, 35(3), 145-154.

Wang, H., Wang, D., \& Peng, Z. (2014). Neural network based adaptive dynamic surface control for cooperative path following of marine surface vehicles via state and output feedback. Neurocomputing, 133, 170-178.

Xiang, X., Lapierre, L., Jouvencel, B., \& Parodi, O. (2009). Coordinated path following control of multiple nonholonomic vehicles. In OCEANS 2009-EUROPE (pp. 1-7). IEEE.

Zhang, F., Fratantoni, D. M., Paley, D. A., Lund, J. M., \& Leonard, N. E. (2007). Control of coordinated patterns for ocean sampling. International Journal of Control, 80(7), 1186-1199.

Zhang, Q., Lapierre, L., \& Xiang, X. (2013). Distributed control of coordinated path tracking for networked nonholonomic mobile vehicles. IEEE Transactions on Industrial Informatics, 9(1), 472-484.

Zhang, F., \& Leonard, N. E. (2007). Coordinated patterns of unit speed particles on a closed curve. Systems \& Control Letters, 56(6), 397-407. 


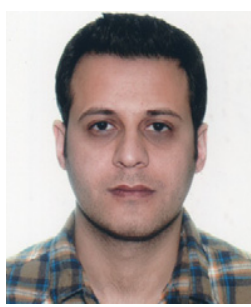

Alireza Doosthoseini received his B.A.Sc. and M.A.Sc. degrees in Mechanical Engineering from the University of Tehran in 2006 and 2009 respectively. He received his Ph.D. degree in Electrical Engineering from the University of Waterloo in 2015. His current research interests are nonlinear control systems, multi-agent systems, and path following.

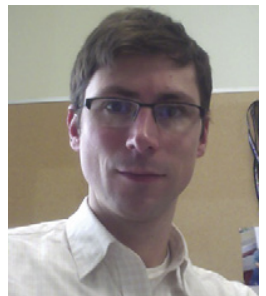

Christopher Nielsen received his B.A.Sc. in Electrical Engineering from the University of Waterloo in 2002. He received his M.A.Sc. and Ph.D. degrees from the University of Toronto in 2004 and 2008 respectively. In 2008 he joined the Department of Electrical and Computer Engineering at the University of Waterloo where he is currently an Associate Professor. His current research interests include geometric control theory, motion control, and output feedback. 